SAND94-1955 • UC-721

Unlimited Release

Printed September 1994

\title{
PP: A Graphics Post-Processor for the EQ6 Reaction Path Code
}

\author{
H. W. Stockman
}

\section{Propared by}

Sandla Natlonal Laboratorios

Albuquerque, Now Moxico 87185 and Livermore, Californla 94550

for the United States Department of Energy

undor Contract DE-AC04-94AL85000

Approved for public relesee; distribution is unlimited. 
Issued by Sandia National Laboratories, operated for the United States Department of Energy by Sandia Corporation.

NOTICE: This report was prepared as an account of work sponsored by an agency of the United States Government. Neither the United States Government nor any agency thereof, nor any of their employees, nor any of their contractors, subcontractors, or their employees, makes any warranty, express or implied, or assumes any legal liability or responsibility for the accuracy, completeness, or usefulness of any information, apparatus, product, or process disclosed, or represents that its use would not infringe privately owned rights. Reference herein to any specific commercial product, process, or service by trade name, trademark, manufacturer, or otherwise, does not necessarily constitute or imply its endorsement, recommendation, or favoring by the United States Government, any agency thereof or any of their contractors or subcontractors. The views and opinions expressed herein do not necessarily state or reflect those of the United States Government, any agency thereof or any of their contractors.

Printed in the United States of America. This report has been reproduced directly from the best available copy.

Available to DOE and DOE contractors from

Office of Scientific and Technical Information

PO Box 62

Oak Ridge, TN 37831

Prices available from (615) 576-8401, FTS 626-8401

Available to the public from

National Technical Information Service

US Department of Commerce

5285 Port Royal Rd

Springfield, VA 22161

NTIS price codes

Printed copy: A03

Microfiche copy: A01 
Distribution

SAND-94-1955

Category UC-721

\author{
Unlimited Release
}

: Printed September 1994

\title{
PP: A GRAPHICS POST-PROCESSOR FOR THE EQ6 REACTION PATH CODE
}

\author{
H.W. Stockman \\ Geochemistry Department 6118 \\ Sandia National Laboratories \\ Albuquerque, NM 87185-0750
}

\begin{abstract}
The PP code is a graphics post-processor and plotting program for EQ6, a popular reaction-path code. PP runs on personal computers, allocates memory dynamically, and can handle very large reaction path runs. Plots of simple variable groups, such as fluid and solid phase composition, can be obtained with as few as two keystrokes. Navigation through the list of reaction path variables is simple and efficient. Graphics files can be exported for inclusion in word processing documents and spreadsheets, and experimental data may be imported and superposed on the reaction path runs. The EQ6 thermodynamic database can be searched from within PP, to simplify interpretation of complex plots.
\end{abstract}




\section{Acknowledgments}

The author thanks Jim Garner and Christine Stockman of Sandia Dept. 6342 for testing the many versions of PP, and Thomas J. Wolery of Lawrence Livermore National Laboratory for helpful advice and the chance to use EQ6. 


\section{DISCLAIMER}

Portions of this document may be illegible in electronic image products. Images are produced from the best available original document. 


\section{Contents}

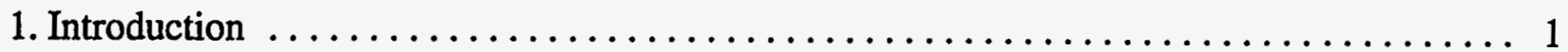

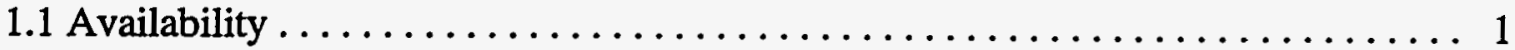

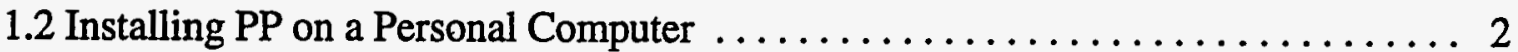

1.3 Starting the Program; Command-line Arguments . . . . . . . . . . . . . 4

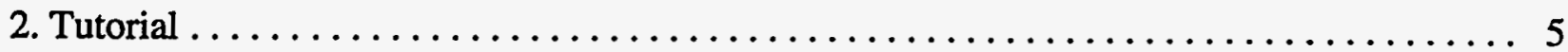

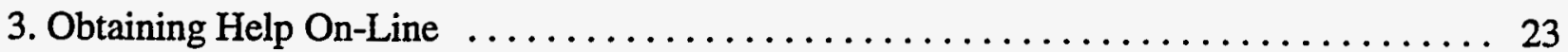

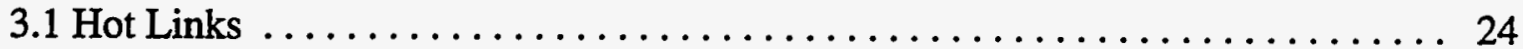

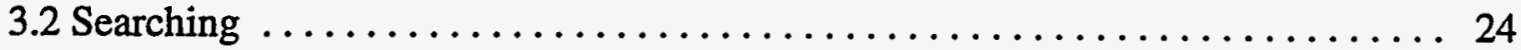

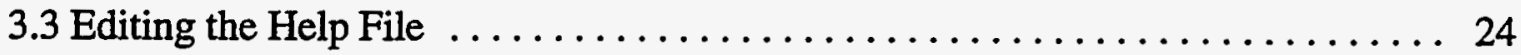

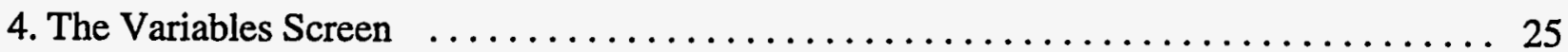

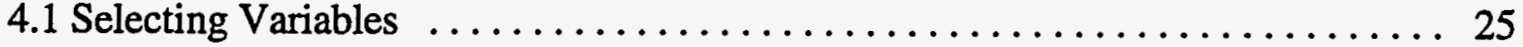

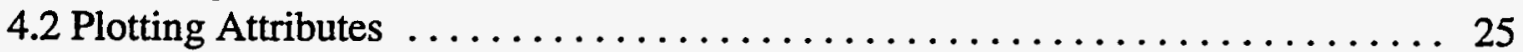

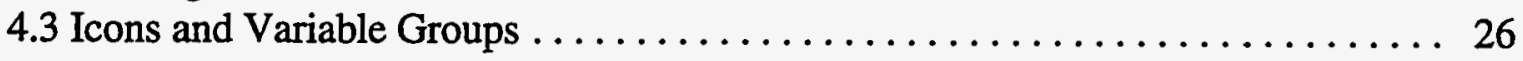

4.4 Buttons (Bottom of Variables Screen) ........................ 27

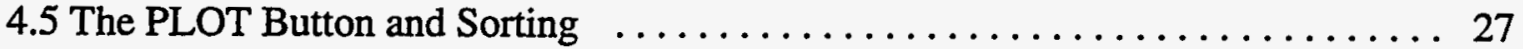

4.6 Templates: Saving (pUt) and Retrieving (Get) Plotting Attributes ......... 27

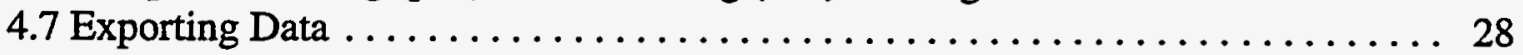

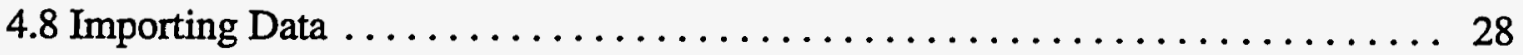

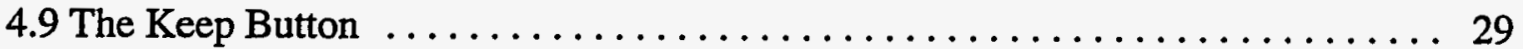

4.10 The mOles / MOLAL Button $\ldots \ldots \ldots \ldots \ldots \ldots \ldots \ldots \ldots \ldots \ldots \ldots, 30$

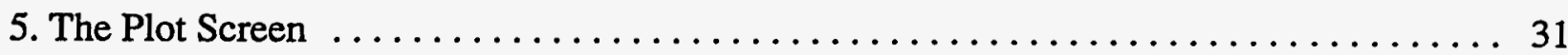

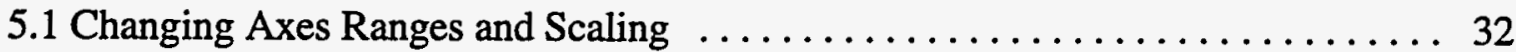

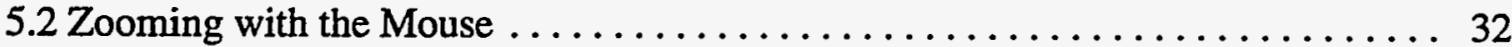

5.3 Changing Curve Labels, Markers (Symbols) and Line Types $\ldots \ldots \ldots \ldots \ldots 32$

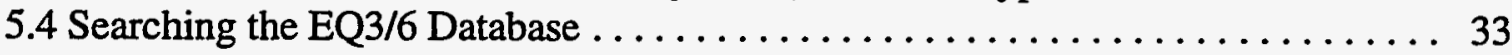

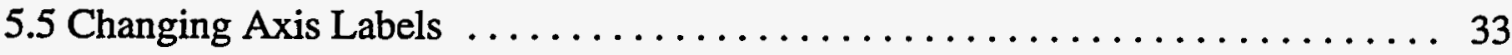

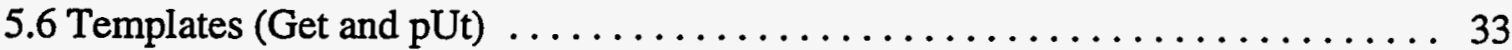

5.7 The Zoom and Label Buttons .............................. 34

5.8 File Options: Output File Characteristics, Plot Appearance $\ldots \ldots \ldots \ldots \ldots \ldots 34$

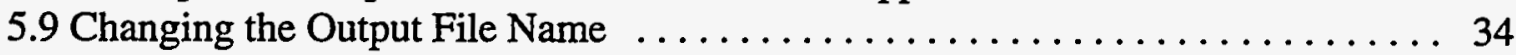

5.10 Changing the Text Font (Plot Screen, File Submenu) ............. 35

5.11 The QA (Quality Assurance) Banner $\ldots \ldots \ldots \ldots \ldots \ldots \ldots \ldots \ldots \ldots \ldots \ldots$

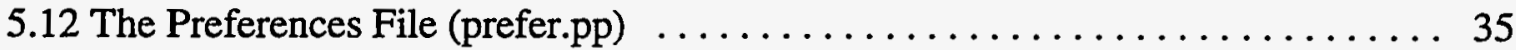

5.13 Output -- Printing, Hardcopy, Importing Plots into Word Processors . . . . . 36

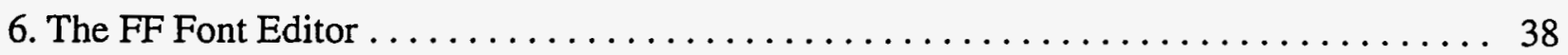

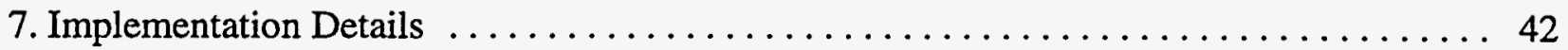




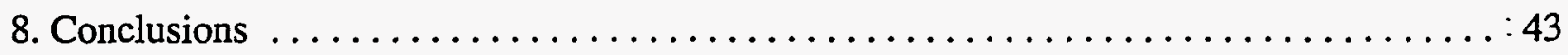

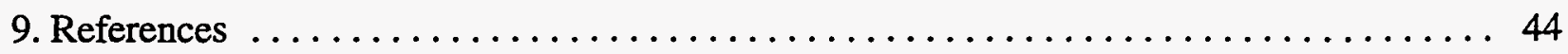

10. Definitions, Copyrights and Trademarks $\ldots \ldots \ldots \ldots \ldots \ldots \ldots \ldots \ldots \ldots \ldots$

APPENDIX: EQ6 Modifications for Creating HWSDATA $\ldots \ldots \ldots \ldots \ldots \ldots \ldots \ldots$

\section{Figures}

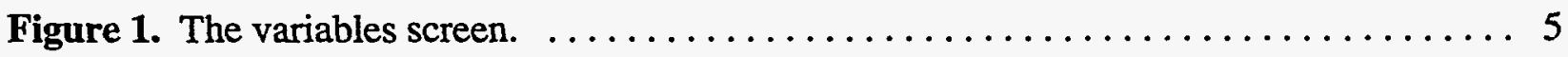

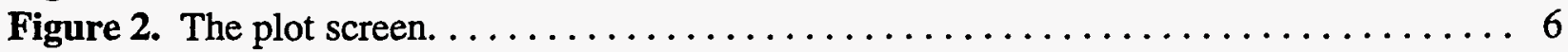

Figure 3. Moving a curve label. ........................... 7

Figure 4. Changing a curve symbol. $\ldots \ldots \ldots \ldots \ldots \ldots \ldots \ldots \ldots \ldots \ldots \ldots \ldots \ldots$

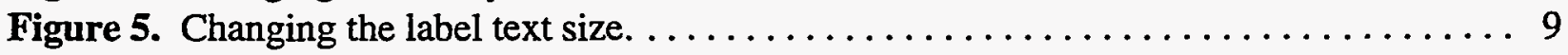

Figure 6. Changing the font from the File options menu. $\ldots \ldots \ldots \ldots \ldots \ldots \ldots \ldots$

Figure 7. Setting a zoom box with the mouse $\ldots \ldots \ldots \ldots \ldots \ldots \ldots \ldots \ldots \ldots \ldots \ldots \ldots \ldots$

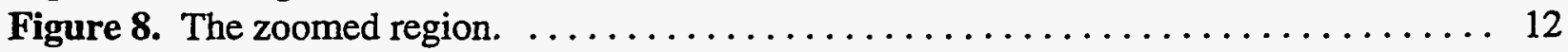

Figure 9. Selecting Al-O species to plot on the right vertical axis. Note ' $\&$ ' can be used to separate word fragments. ................................ 13

Figure 10. Plotting different data types against the left and right vertical axes. . . . . . . 14

Figure 11. Setting axis ranges with the Axes dialog. $\ldots \ldots \ldots \ldots \ldots \ldots \ldots \ldots \ldots \ldots$

Figure 12. PP graphics file hp.000, imported into WordPerfect. . . . . . . . . . . . 16

Figure 13. Selecting imported data. $\ldots \ldots \ldots \ldots \ldots \ldots \ldots \ldots \ldots \ldots \ldots \ldots \ldots \ldots \ldots \ldots \ldots \ldots$

Figure 14. Selecting variables at the top of the list. $\ldots \ldots \ldots \ldots \ldots \ldots \ldots \ldots \ldots \ldots \ldots \ldots$

Figure 15. Editing a label. ............................... 19

Figure 16. Graphics file from figure 15 , imported into WordPerfect. ........... 20

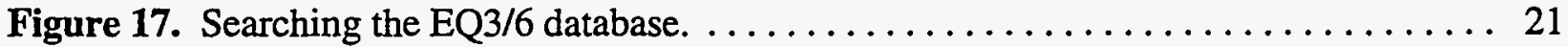

Figure 18. CONTENTS mode for searching the EQ3/6 database. $\ldots \ldots \ldots \ldots \ldots \ldots 22$

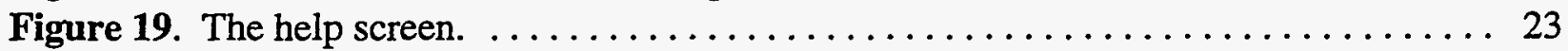

Figure 20. Using FF to edit the letter ' $Q$ '. A right mouse click has selected the nearest polyline for editing. ..................................... 39

Figure 21. Using FF to edit a custom symbol in the font file (external.fnt). ......... 40

Figure 22. Using custom symbols in a plot. ........................ 41 


\section{Introduction}

The EQ6 reaction path code (Wolery, 1992) uses thermodynamic and kinetic data to predict the chemical evolution of systems containing water, rocks and soils, and man-made materials (Bruton and Shaw, 1988; Gardiner et al., 1989; Criscenti and Arthur, 1991). EQ6 is a powerful and flexible tool, but like any reaction path code, it tends to overwhelm the user with information. A single reaction path run may generate concentration and saturation state data for hundreds of aqueous species and mineral phases, sampled over several hundred steps. The ASCII tables produced by EQ6 are well-organized, and generally sample only the most significant portions of the reaction path (typically fewer than $20 \%$ of the calculated steps); nonetheless, these tables can be several megabytes in size, and analysis of the results can be an intimidating prospect for all but the very experienced user. Increasingly, EQ6 is applied to problems of waste disposal and environmental modeling, and the calculations must be performed by users with little experience in reaction path modeling or computational chemistry.

The program PP is a simple but flexible graphics post-processor for EQ6. PP provides a "point and click" environment for plotting the results of reaction path runs, for exporting the results to spreadsheet files, $\mathrm{CAD}$ and word processing programs, and for exploring the EQ6 thermodynamic databases. PP was written to make EQ6 accessible to a wider range of investigators, particularly "occasional" users with some understanding of aqueous chemistry, but modest knowledge of the computing environment. By using a compact binary input file, PP can preserve data from all steps calculated by EQ6. This report provides instructions for installing $\mathrm{PP}$ on an personal computer, a tutorial, and documentation of more advanced capabilities.

\subsection{Availability}

The binaries for PP are available from the author (e-mail hwstock@sandia.gov), subject to the constraints indicated in the closing banner of the program, viz:

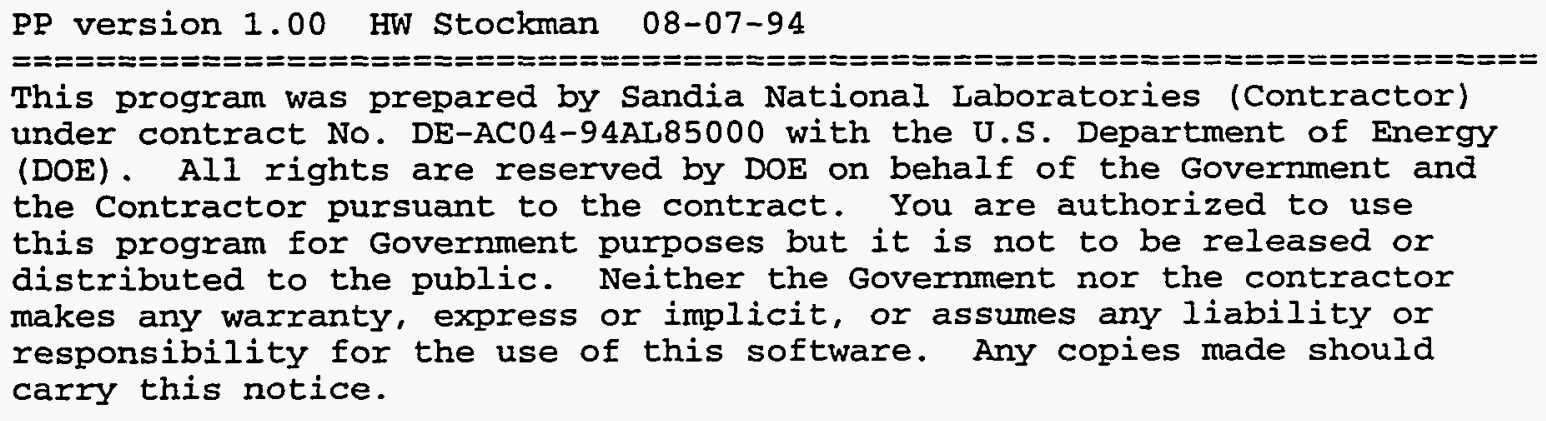

The EQ3/6 code suite is copyrighted by the University of California, and is independent of PP. Instructions for obtaining EQ3/6 can be found in the EQ3/6 manuals (Wolery, 1992). Currently, the Energy Science and Technology Software Center in Oak Ridge, TN (615-576-2606) handles distribution of EQ3/6. The appendix of this report describes the function that must be added to EQ6 to produce the binary plot files read by PP. 


\subsection{Installing PP on a Personal Computer}

PP runs under DOS on "IBM-compatible" computers based on Intel 486DX, 486SL or Pentium, and compatible processors. The computer should have at least $4 \mathrm{MB}$ of free hard disk space and $2 \mathrm{MB}$ of RAM, and a video adapter (graphics card) capable of supporting at least 16 colors at a resolution $640 \times 480$ pixels. The program will run on 386 -based computers with an $80 \times 87$ compatible math coprocessor, but performance may be unacceptable. If $\mathrm{PP}$ is used to search the EQ6 databases, the computer should have at least $4 \mathrm{MB}$, and preferably $8 \mathrm{MB}$ RAM. PP contains its own DOS-extender, runs in 386 protected mode, and allocates memory dynamically as needed. PP can also run full-screen under Microsoft Windows 3.1, and several copies of the program can be active at once, operating on different data sets.

To install PP, first create a directory called $\backslash \mathrm{pp}$ on the hard drive by typing the following sequence of commands at the DOS prompt:

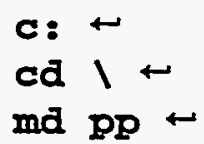

where the " $"$ represents the "Enter" key. Then enter the directory by typing:

$c d p p \leftarrow$

Now copy the distribution file onto the hard disk:

copy a:pp4eq6.exe c:lpp

If the floppy disk drive is called $\mathbf{b}:$, substitute $\mathbf{b}:$ for $\mathbf{a}$ : above. Then type:

pp4eq6 -

which will extract the following files:

\begin{tabular}{|c|c|}
\hline pp.exe & -- the main program; \\
\hline IP_pp & -- the on-line help file (section 3.3); \\
\hline external. fnt & -- the "external" or triplex font file (section 5.10); \\
\hline ff.exe & -- a simple font editor (section 5.10); \\
\hline hwsdata & -- a sample data file, from the EQ6 micro. $6 i$ run; \\
\hline eq6_mods.: & $\begin{array}{c}\text {-- eq6. for modifications necessary to produce the } \\
\text { hwsdata binary file (Appendix); and }\end{array}$ \\
\hline & -- EQ6 input files for second part of tutorial. \\
\hline
\end{tabular}


To help PP find help_pp and the external. fnt, add the following line to the autoexrec.bat file:

set $\mathrm{PP}=\mathrm{C}: \backslash \mathrm{PP}$

Note it is important to capitalize "PP=".

Like most reaction path codes, EQ6 uses mineral names to identify solid phases. Sometimes these names are quite peculiar (e.g., mirabelite and haiweeite), and give no hint about the composition of the phase. PP has a facility to locate the composition, thermodynamic and reference data for these minerals, by searching the EQ3/6 database. To search the EQ3/6 database through $\mathrm{PP}$, one should also set the $\mathrm{EQ36} \_\mathrm{DB}$ environment variable to point to the directory where the EQ3/6 data . * files are stored. That is, a statement such as

\section{set $E Q 36 \_D B=[$ drive $]: \backslash[$ path $]$}

should be added to autoexec.bat. Typically the path will be leq3_6v7.2a limport (see also Searching the EQ3/6 database, section 5.4).

Finally, add Ipp to the path statement in autoexec .bat, so the computer will be able to find the executable files regardless of the working directory. 


\subsection{Starting the Program; Command-line Arguments}

PP can be started ("invoked") by typing pp $\leftarrow$ at the DOS prompt. However, PP can also be invoked with one or two optional command-line parameters:

\section{pp $[6|L| 8 \mid M] \quad[f i l e n a m e] \leftarrow$}

The order of the two parameters is unimportant; that is, [filename] can appear first.

The $[6|\mathrm{~L}| \mathbf{8} \mid \mathrm{M}]$ argument attempts to force a particular video mode. The "8" or " $\mathrm{M}$ " selection tries to force medium resolution, $800 \times 600$ pixel "superVGA" mode; this is the preferred mode, striking a balance between speed and resolution. The "6" or " $L$ " selection tries to force low-resolution $640 \times 480$ pixel mode. If there is no $[6|\mathrm{~L} / 8| \mathrm{M}]$ parameter, PP will attempt to pick the highest resolution supported by the computer's video hardware. Some LAP-TOP computers require the "6" switch; if PP is allowed to pick the "best" video mode, the screen may appear totally blank. If the computer has a VESA driver, it is often helpful to load this driver before running PP (consult the video card documentation for the name and location of the VESA driver; the name often contains the word "vesa"). Since graphics card technology is changing rapidly, it is safest to begin with the "pp 6" invocation. If PP comes up with an unreadable screen, press the Esc key, then the "Y" key to return to DOS.

The [filename] argument specifies an alternate name for the binary input file. The modified version of EQ6 (Appendix) produces a binary file named hwsdata, and by default PP looks for that file in the current directory. Each successive EQ6 run destroys the old hwsdata file. It may be desirable to save the results of previous runs by renaming the hwsdata files, or storing them in another location. The [filename] option makes it possible for PP to read these renamed files.

Here are some sample invocations and corresponding actions:
pp $\leftarrow$
PP reads hwsdata, starts at highest possible resolution;
pp $8 \leftarrow$
PP reads hwsdata, starts at $800 \times 600$ pixel resolution;
pp 6 hwsdata.0ld $\leftarrow$ PP reads hwsdata.01d, starts at 640x480 pixel resolution;
$p p$ hwsdata.old I $\leftarrow$ Same as previous line $(6=\mathrm{L}$, order unimportant).

PP has only two main screens: (1) the variables screen, entered first, which lists all the variables calculated by EQ6; and (2) the plot screen, which allows one to see, edit, and output x-y plots of elemental, mineral, fugacity and species concentration or saturation states. Both screens are mouse-aware, though one can perform nearly all actions with the keyboard by pressing the "hot keys" on the menu bars. Mouse-aware menu bars have a dark cyan (blue-green) background, with light cyan buttons (highlights). The hot keys are indicated by bright white letters on a dark red or green background. NOTE that one moves back and forth between the screens with the "PLOT" and "Varia" buttons. To exit the program, press the Esc key and then type 'Y'. The Esc key can also be used to "back out" from most of PP's menus and dialog boxes. 


\section{Tutorial}

To begin the tutorial, switch to the directory that contains the hwsdata file and invoke PP by typing pp $8 \leftarrow$ (preferred) or pp $6 \leftarrow$ at the DOS prompt.

PP will come up in the variables screen. A small arrow (the mouse cursor will move about the screen as the mouse is moved.

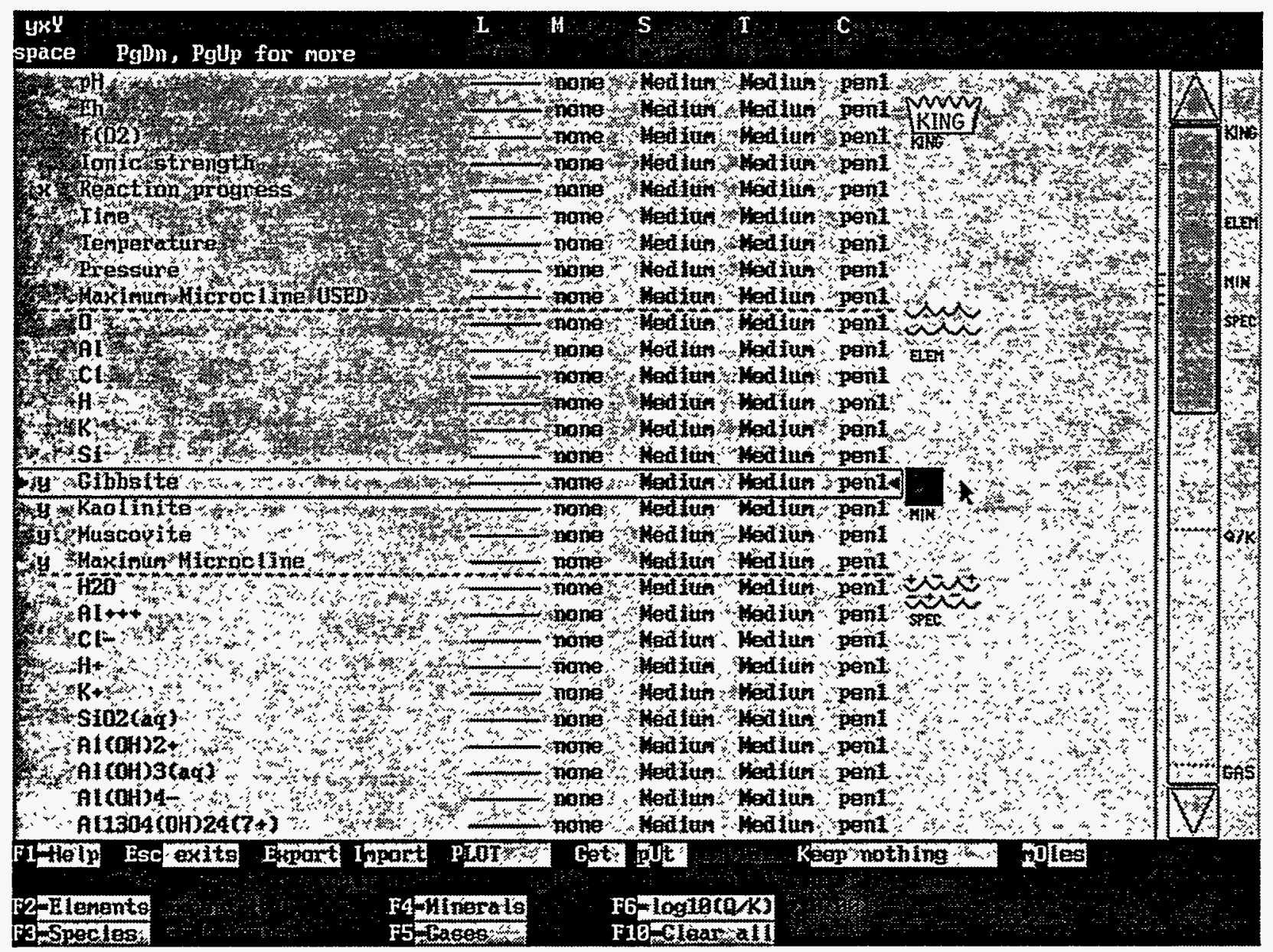

Figure 1. The variables screen.

Move the mouse cursor over the "minerals" icon law and click the left mouse button. A lowercase ' $y$ ' will appear in the left-most column next to each mineral name, indicating that these variables have been selected for plotting on the left vertical axis (figure 1). If the right mouse button were clicked instead, uppercase 'Y's would appear, indicating the variables had been chosen to plot against the right vertical axis. If the computer lacks a mouse, press F4 to select all the minerals. 


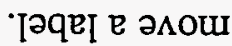

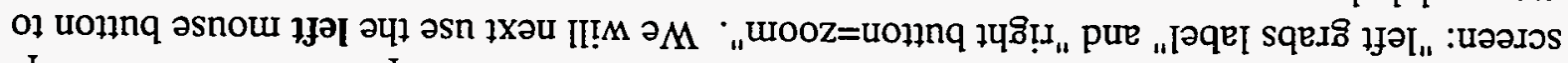

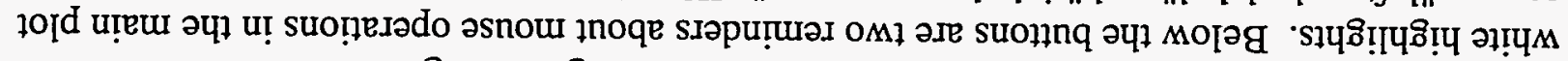

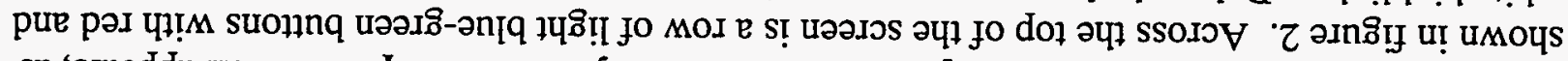

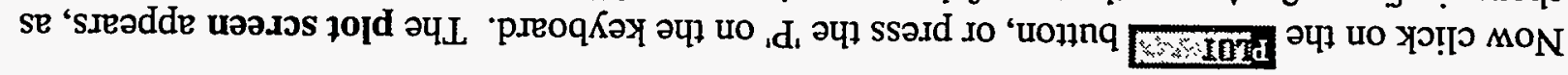

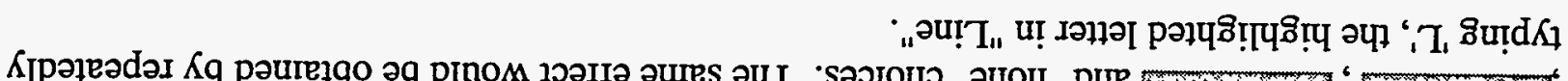

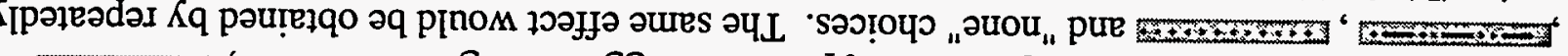

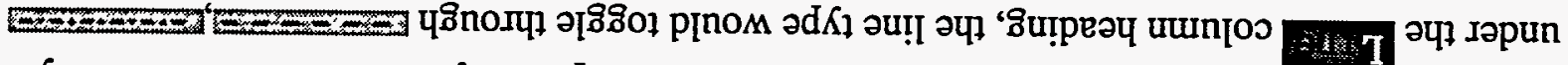

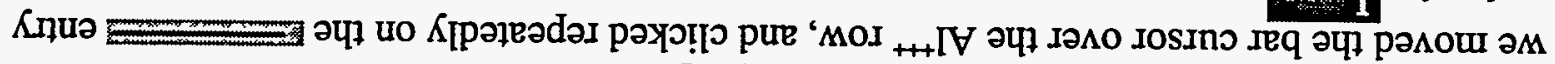
J! snuL

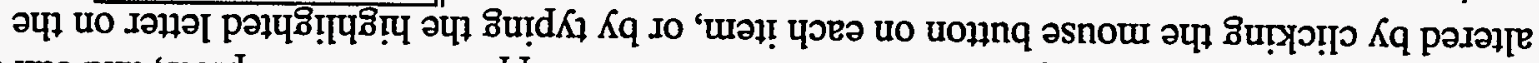

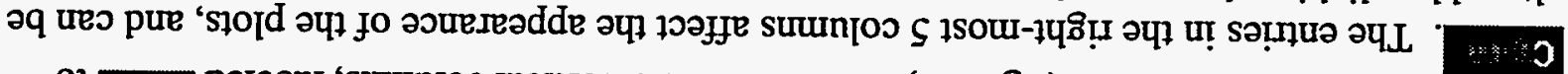

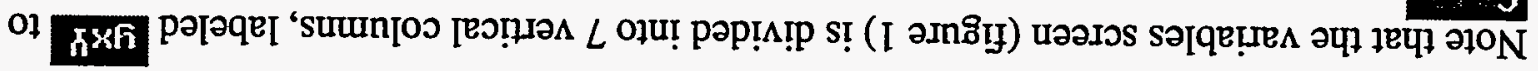

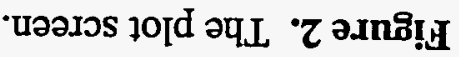

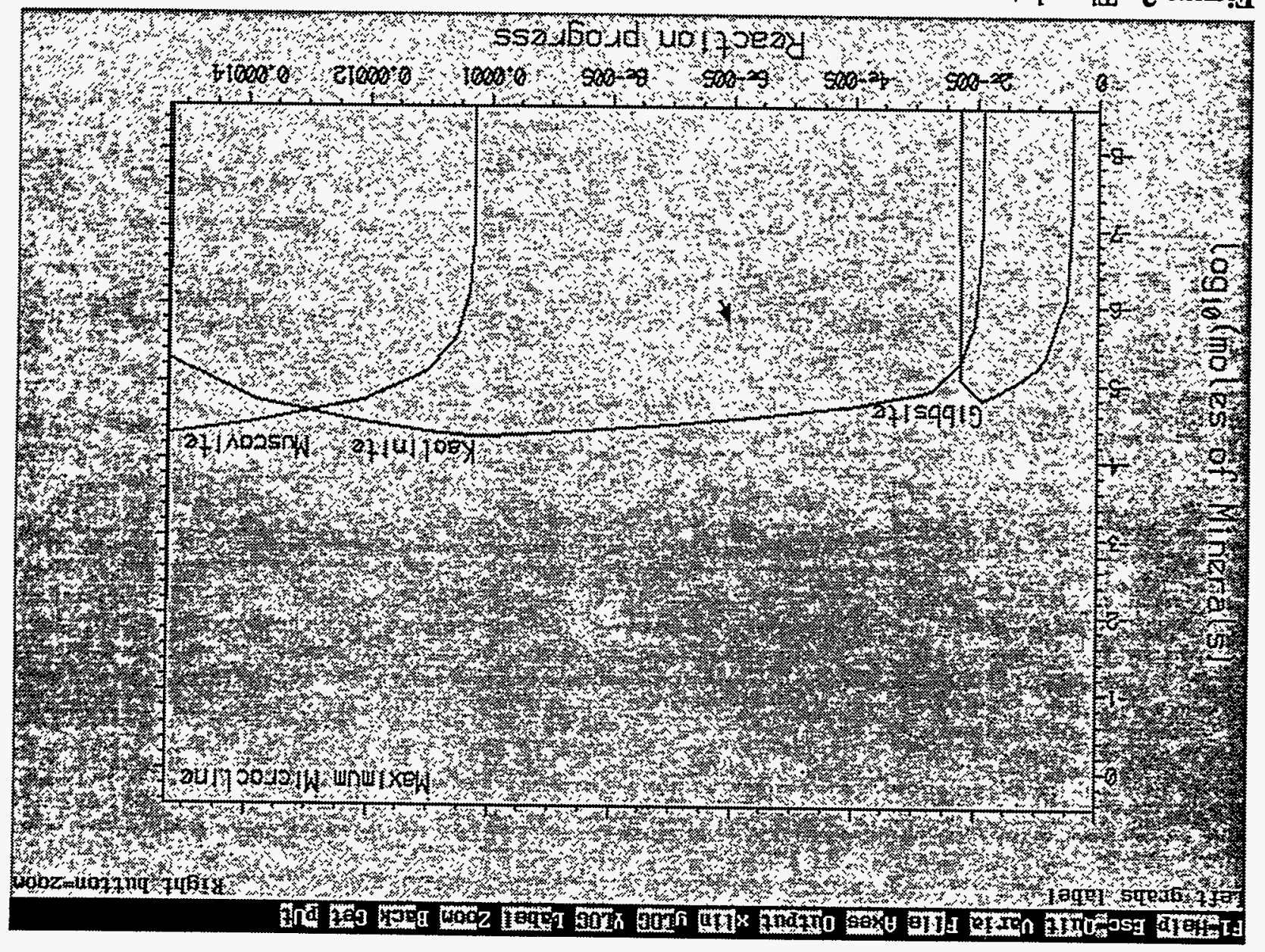




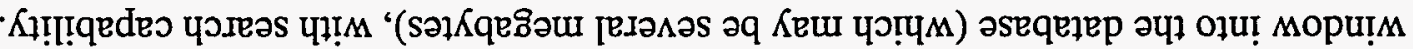

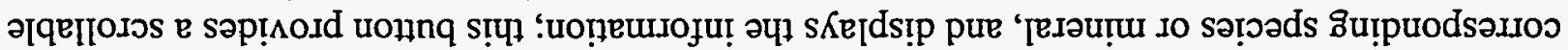

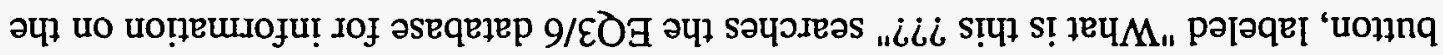

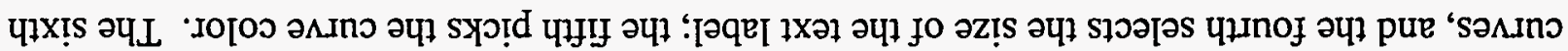

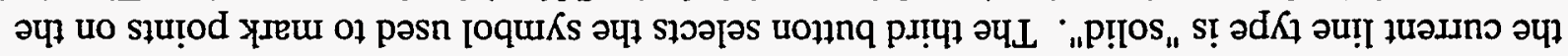

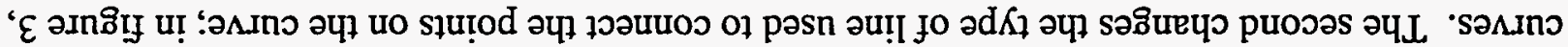

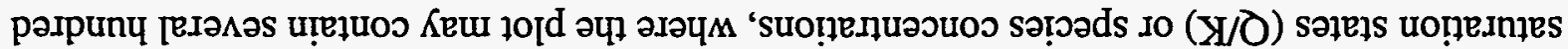

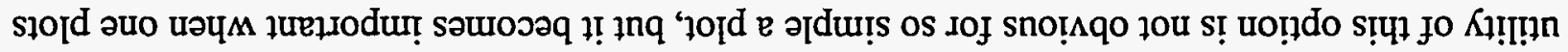

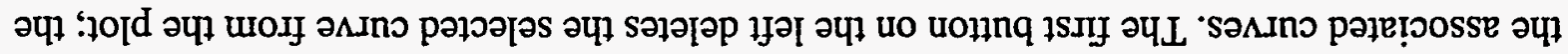

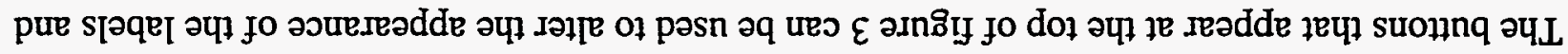

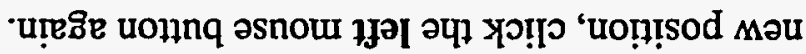

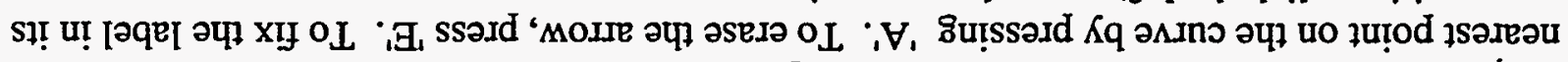

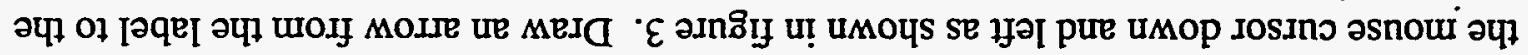

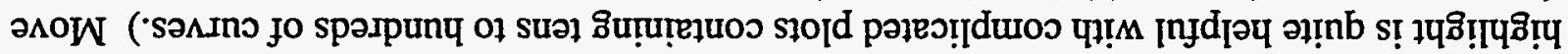

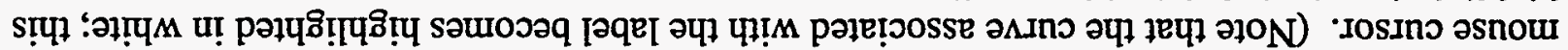

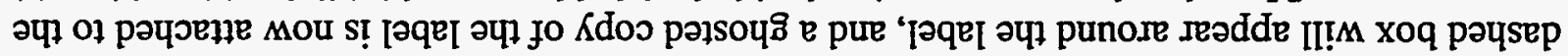

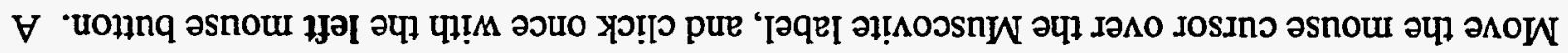

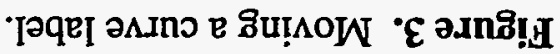

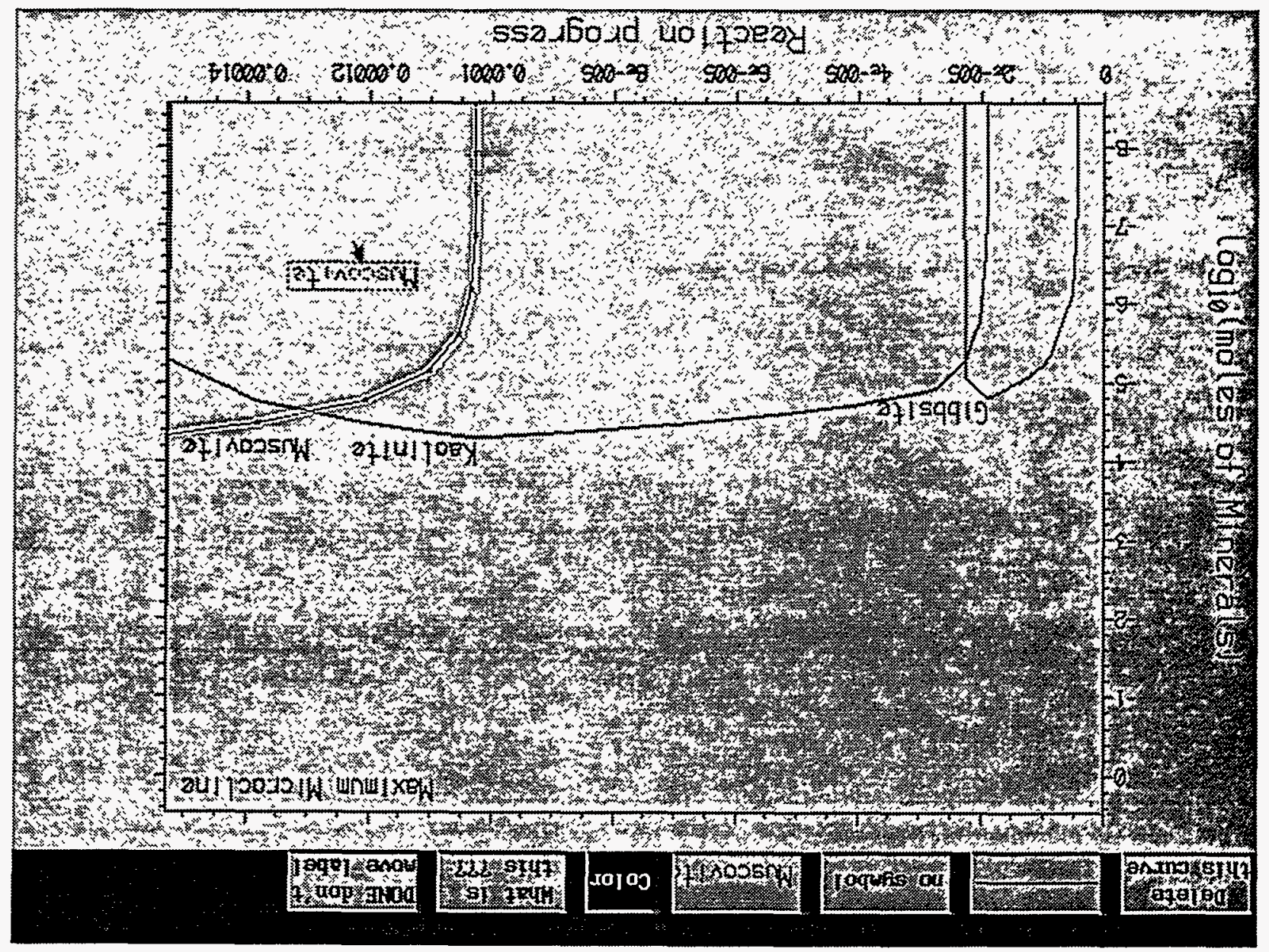


-uonisod

(sno!̣

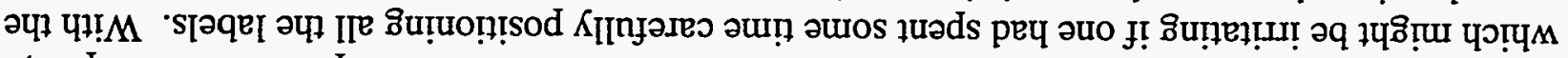

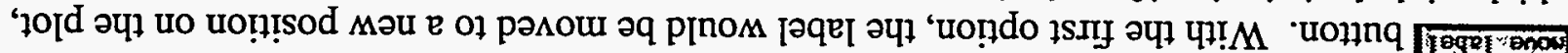

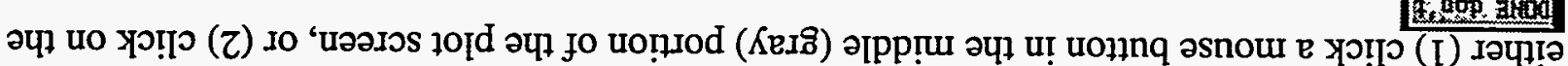

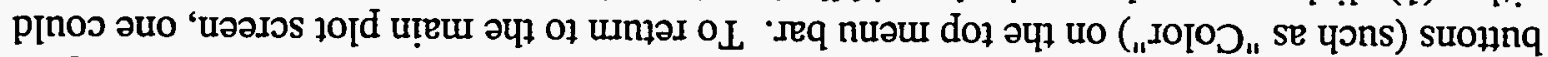

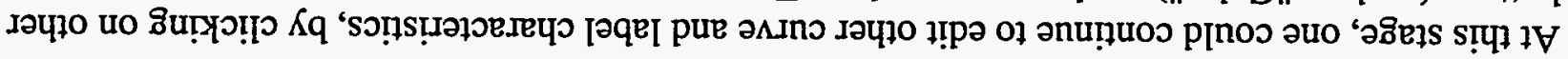

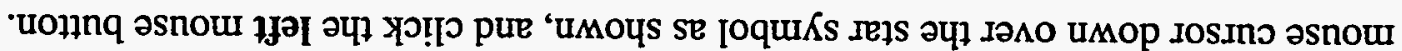

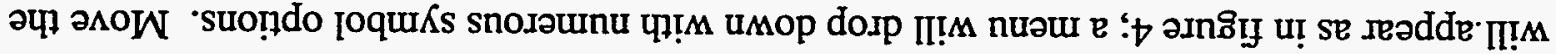

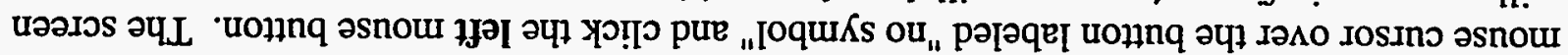

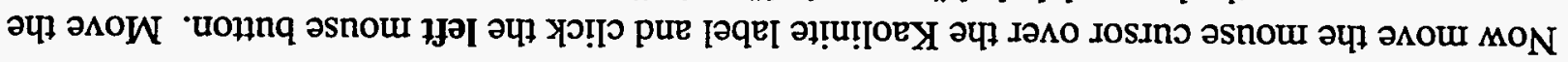

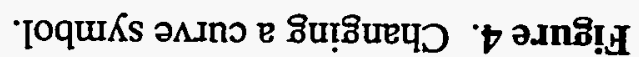

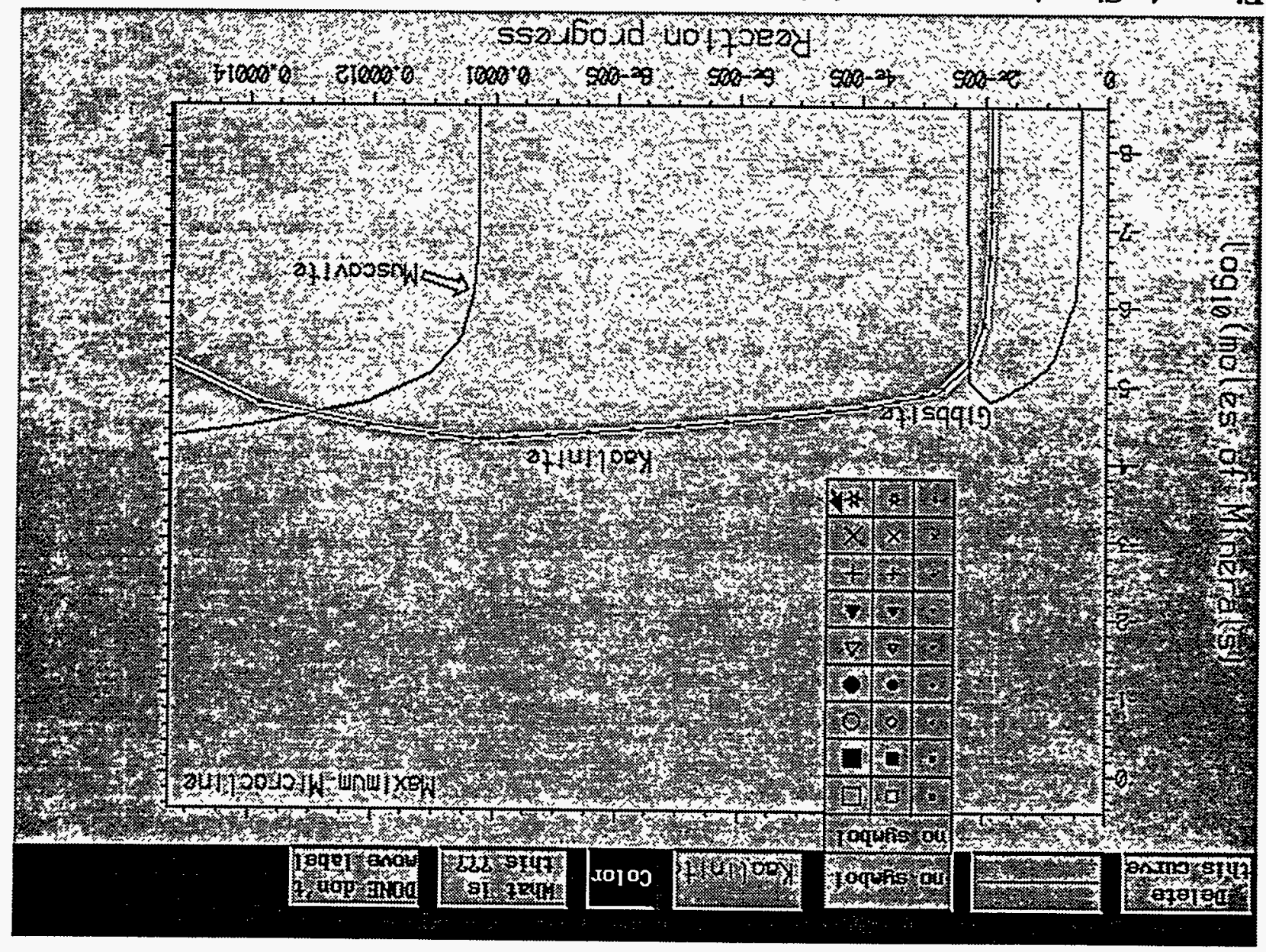




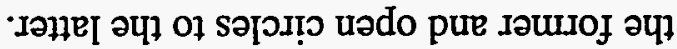
ol s[oqu $K s$ әrenbs pəII

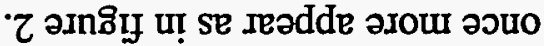

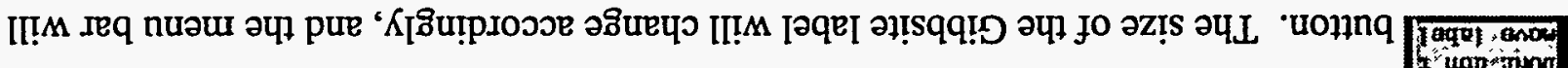

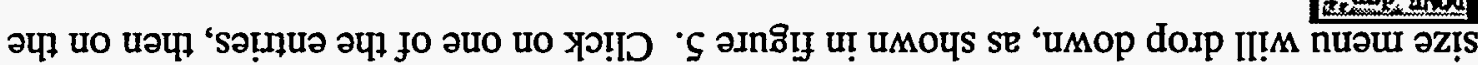

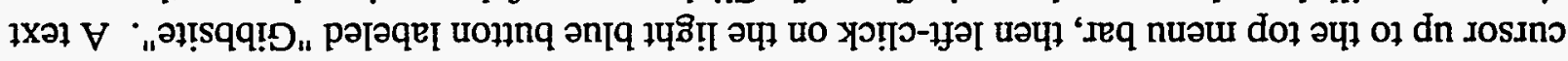

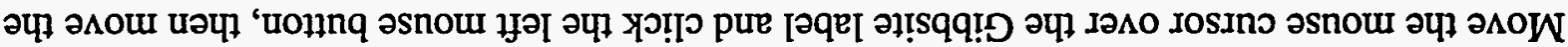

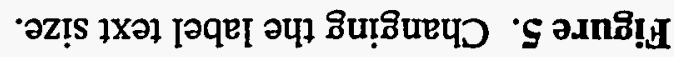

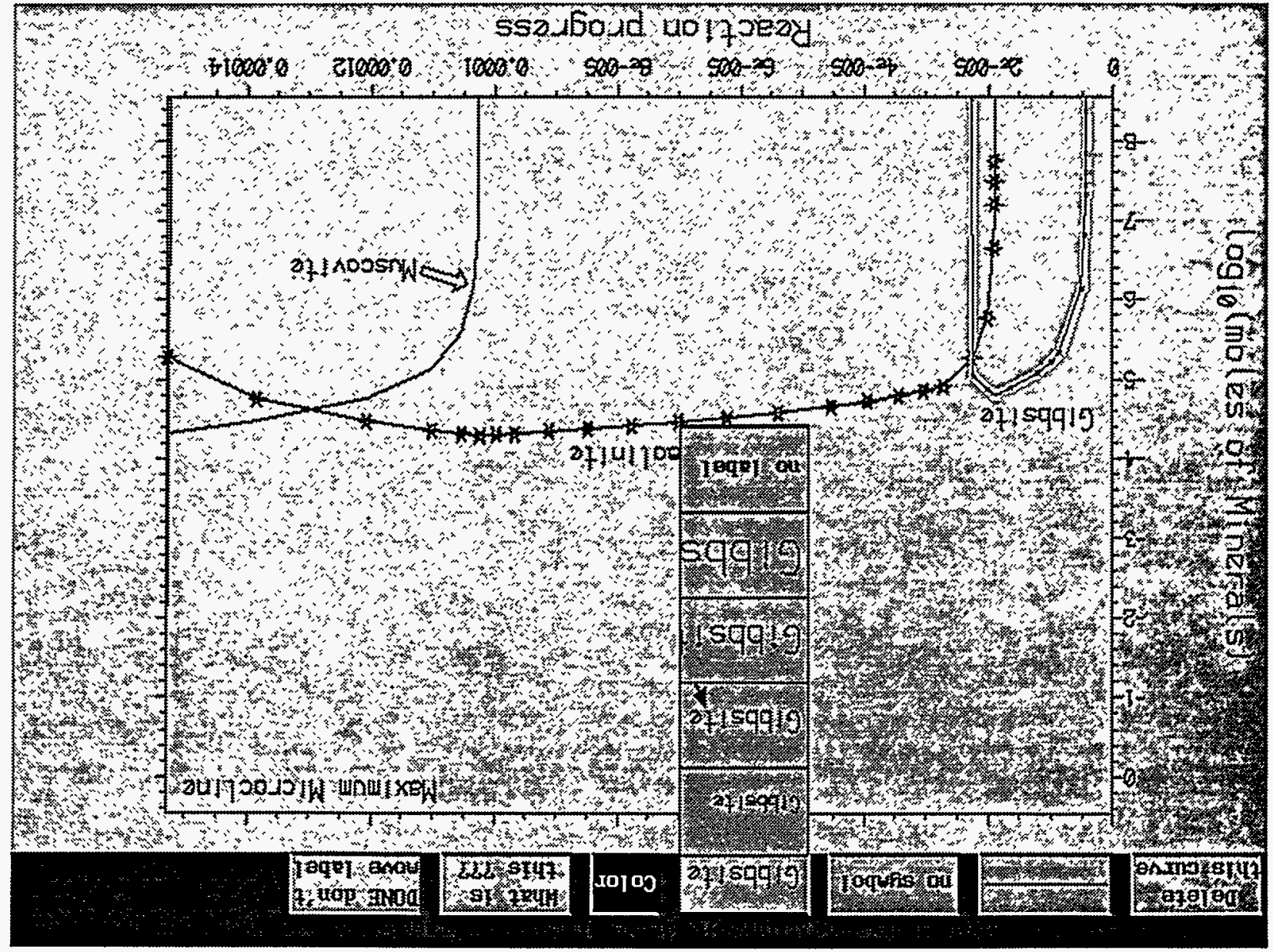




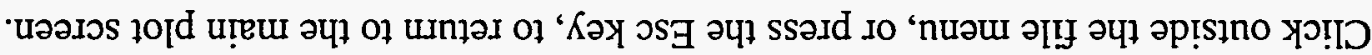

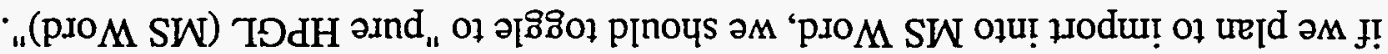

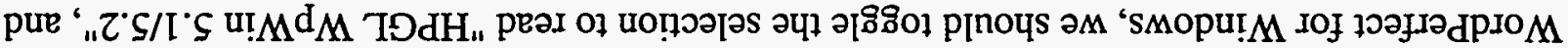

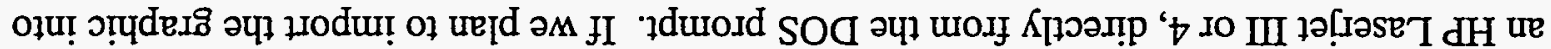

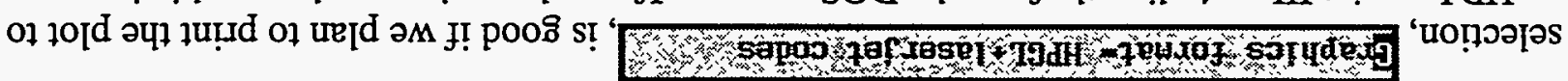

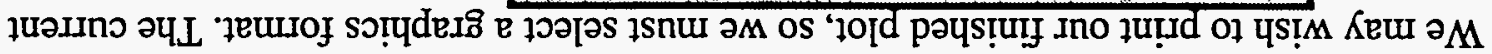

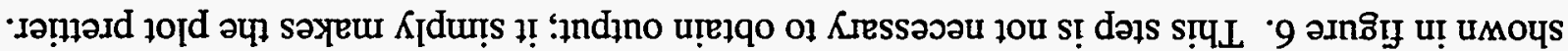

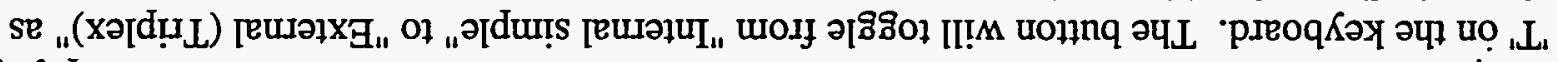

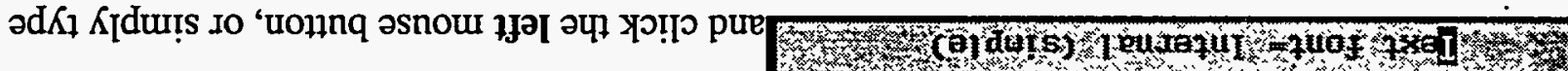

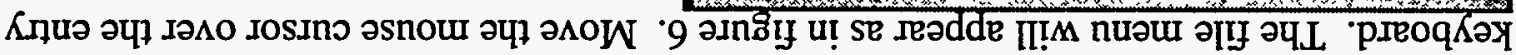
әчl uo ،

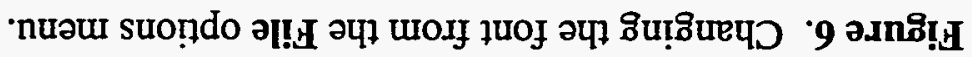

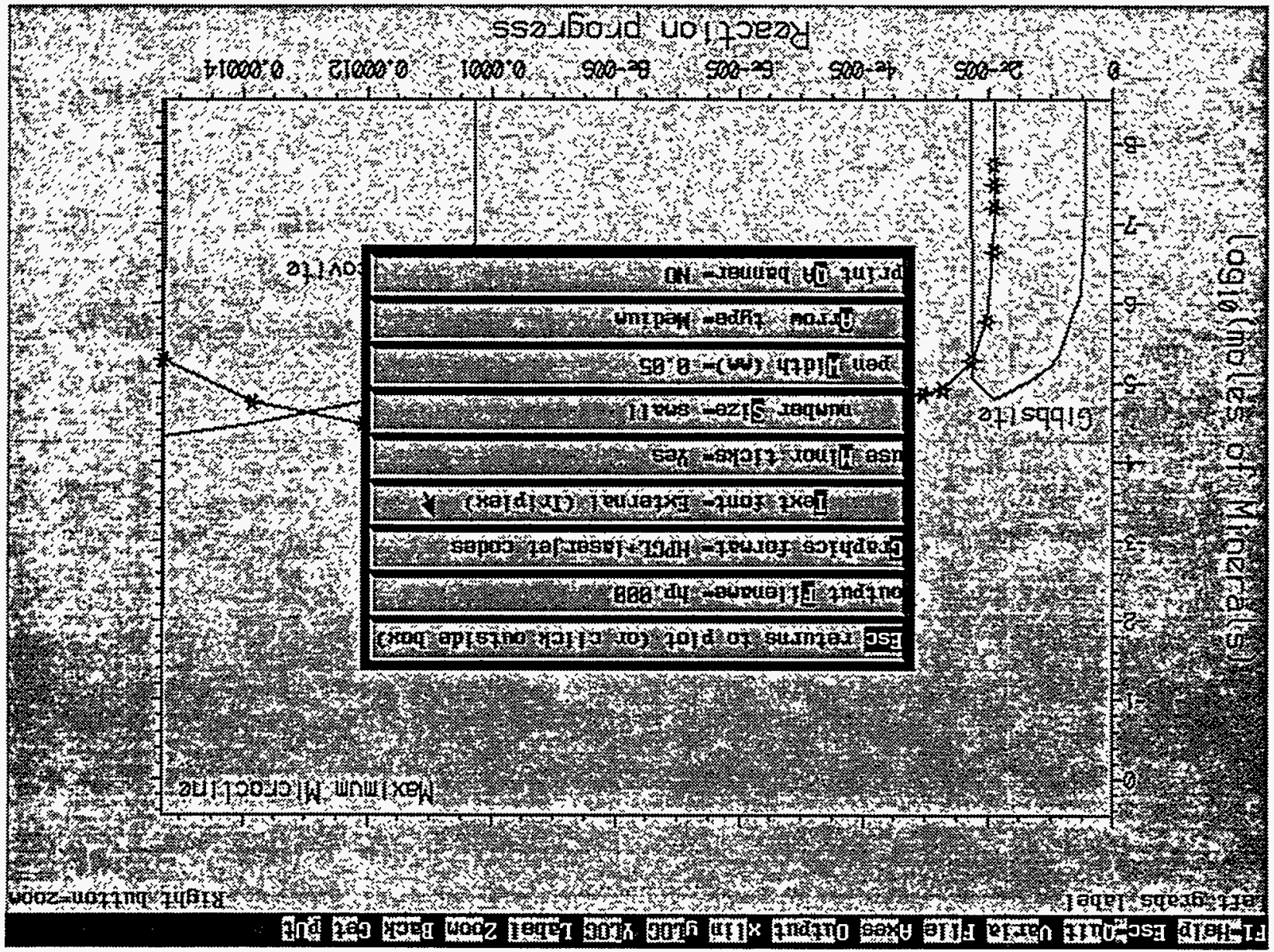




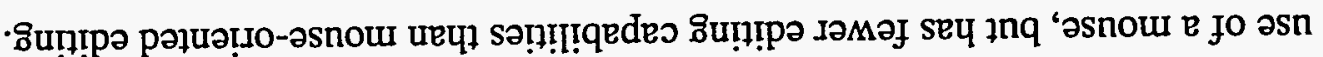

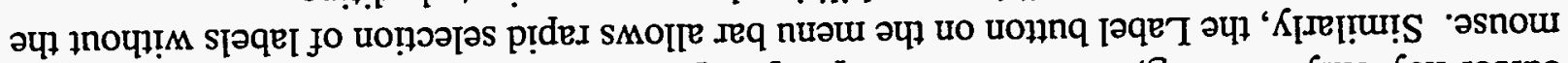

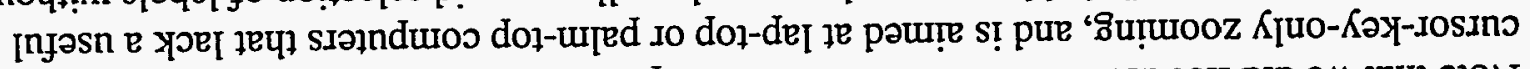

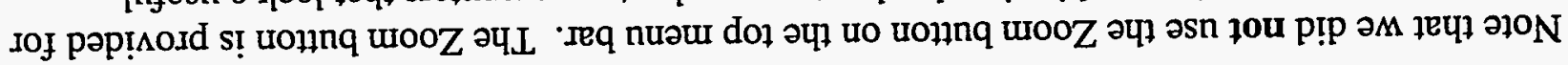

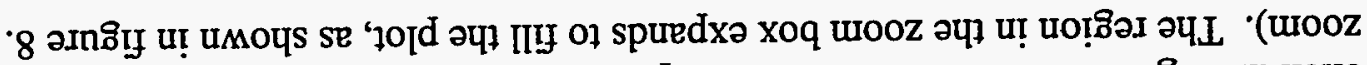

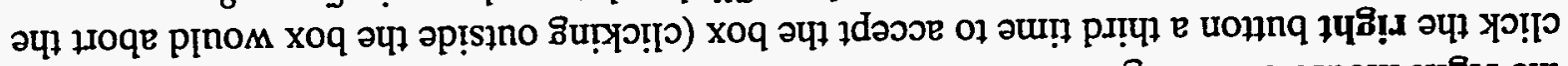

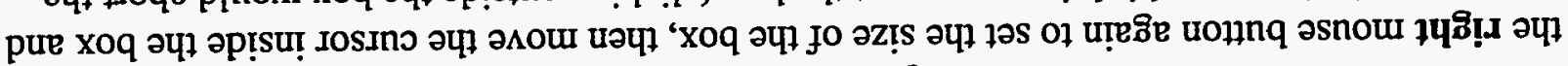

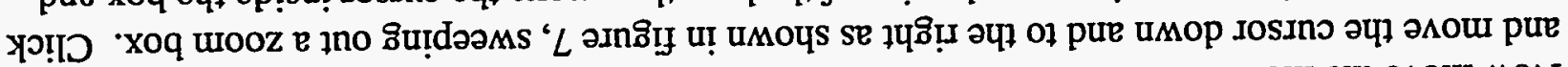

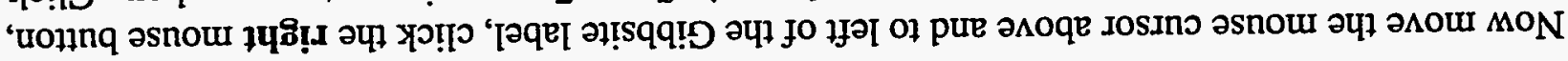

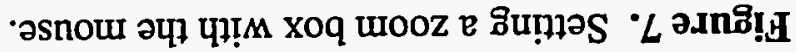

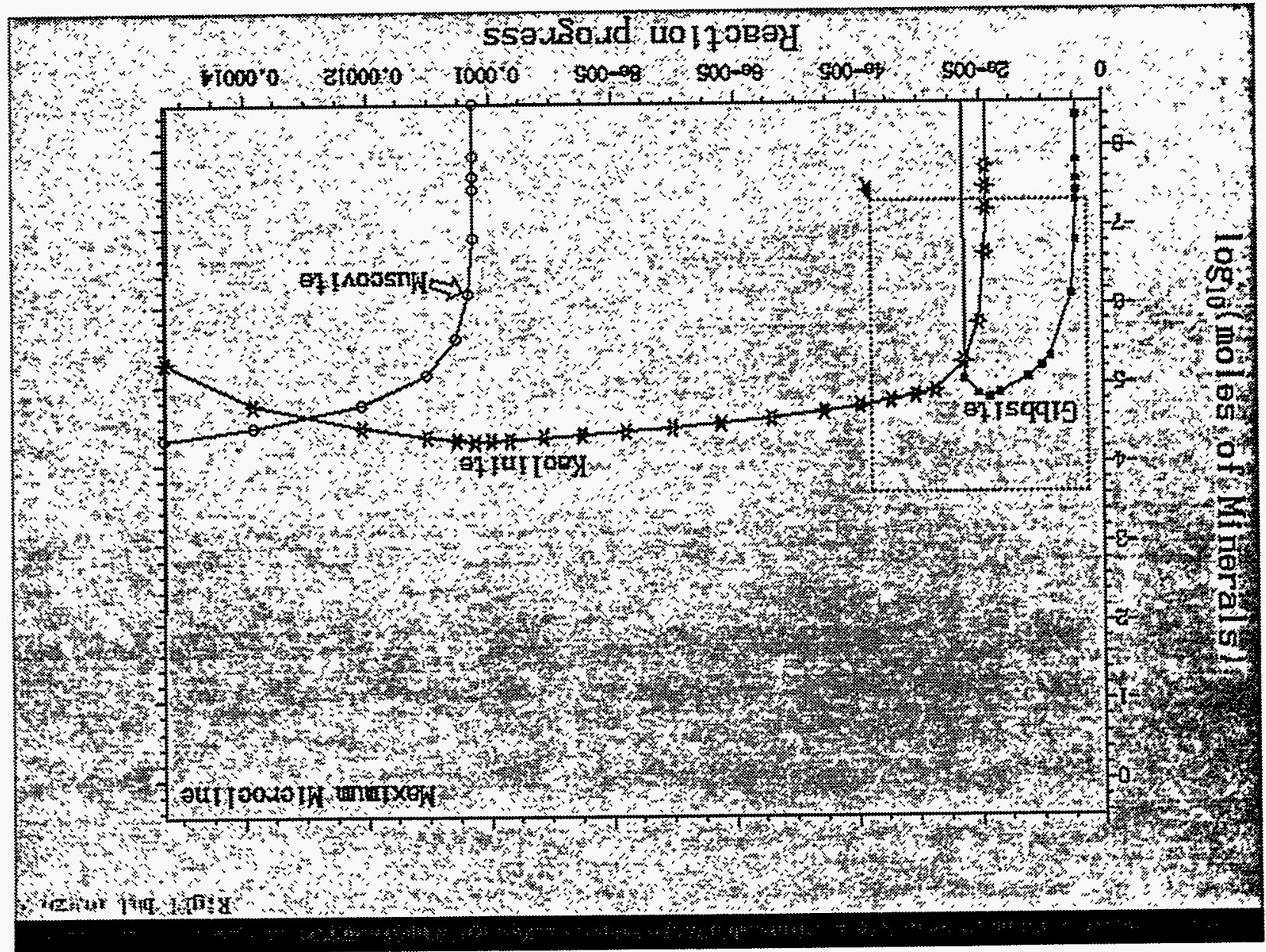




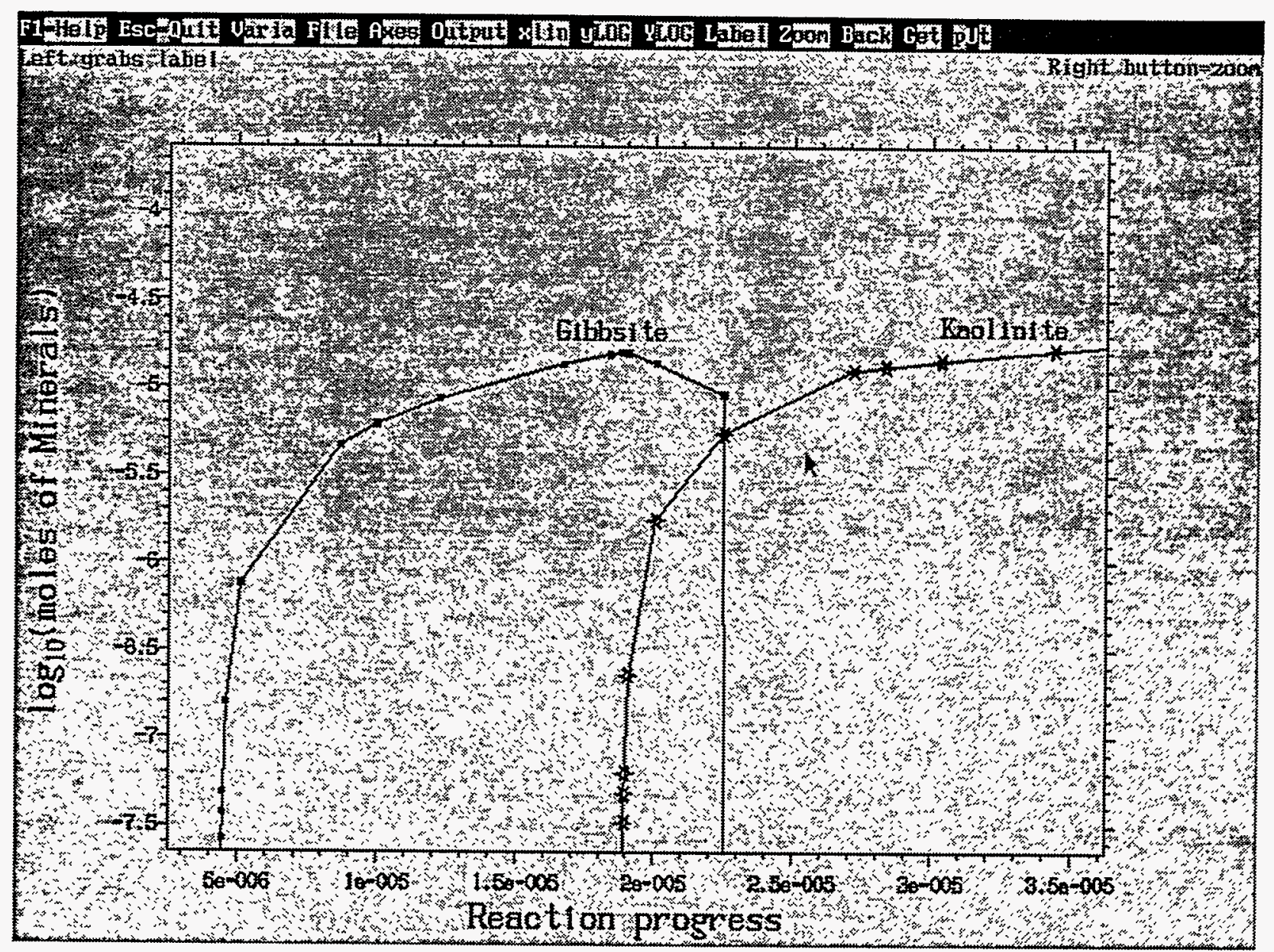

Figure 8. The zoomed region.

Now restore the previous scaling by clicking on the $\mathrm{Back}$ button, or by pressing ' $\mathrm{B}$ ' on the keyboard. Then return to the variables screen by clicking on the Virita button, or by typing ' $V$ ' on the keyboard.

In the plot so far, the mineral amounts are plotted against the left vertical axis. We now wish to plot the molalities of aluminum species, containing both $\mathrm{Al}$ and oxygen, against the right vertical axis. 


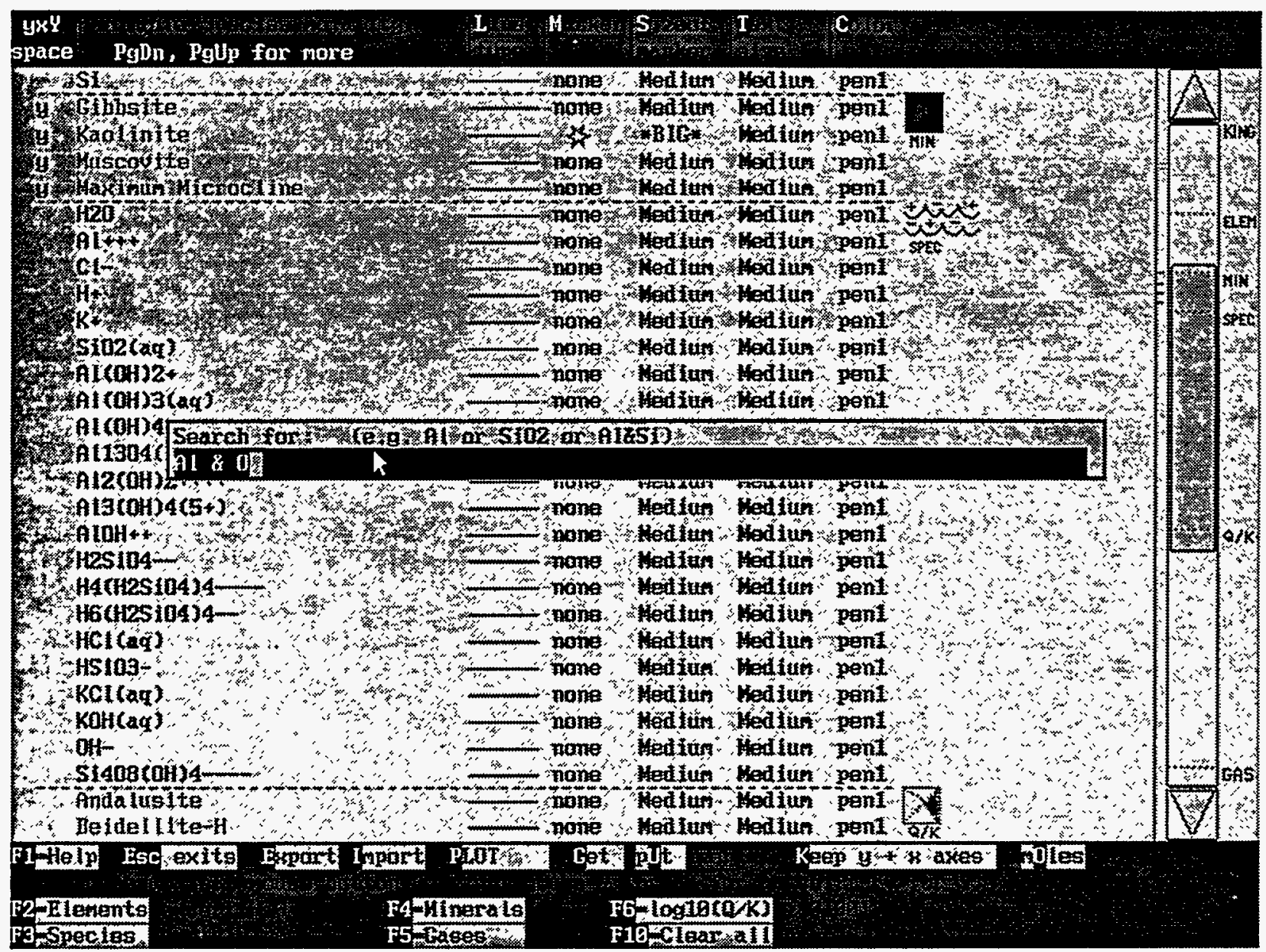

Figure 9. Selecting Al-O species to plot on the right vertical axis. Note ' $\&$ ' can be used to separate word fragments.

First grab the slider of the scroll bar, and drag it down so it straddles the region between "SPEC" and "Q/K". To do so, position the mouse cursor over the bright blue slider on the right side of the screen, depress the left mouse button, and hold the button down while moving the slider to its new position, then release the mouse button. This step is not absolutely necessary, but makes it easier to see the changes.

Next click on the species icon keyboard). A dialog box comes up shown in figure 9, requesting word fragments for the search. Type in "Al \& O", then click outside the dialog box or press the Enter key. Species containing both $\mathrm{Al}$ and $\mathrm{O}$ will be marked in the left-most column with an uppercase ' $\mathrm{Y}$ ', indicating they are to be plotted against the right vertical axis.

Notice the Keep y $y$ axes., which shows PP's intentions to preserve the current $x$ and $y$ axis scaling. We could override this choice, and force PP to rescale the axes, by clicking 3 times on the button, until it reads "Keep nothing" (if you try that, click another 4 times to return to "Keep y + $\mathrm{x}$ axes" before proceeding). 


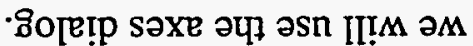

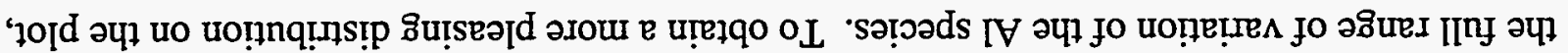

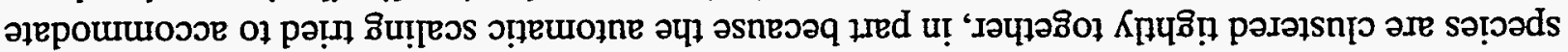

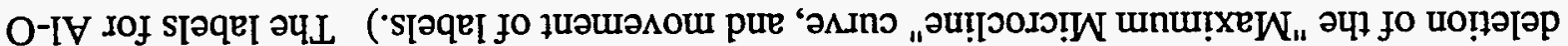

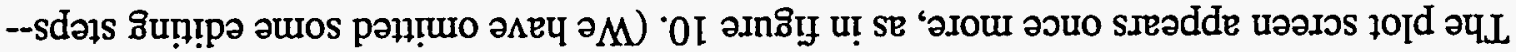

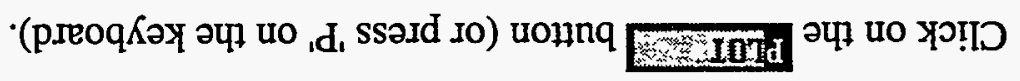

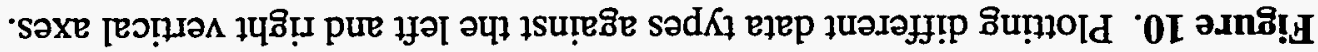

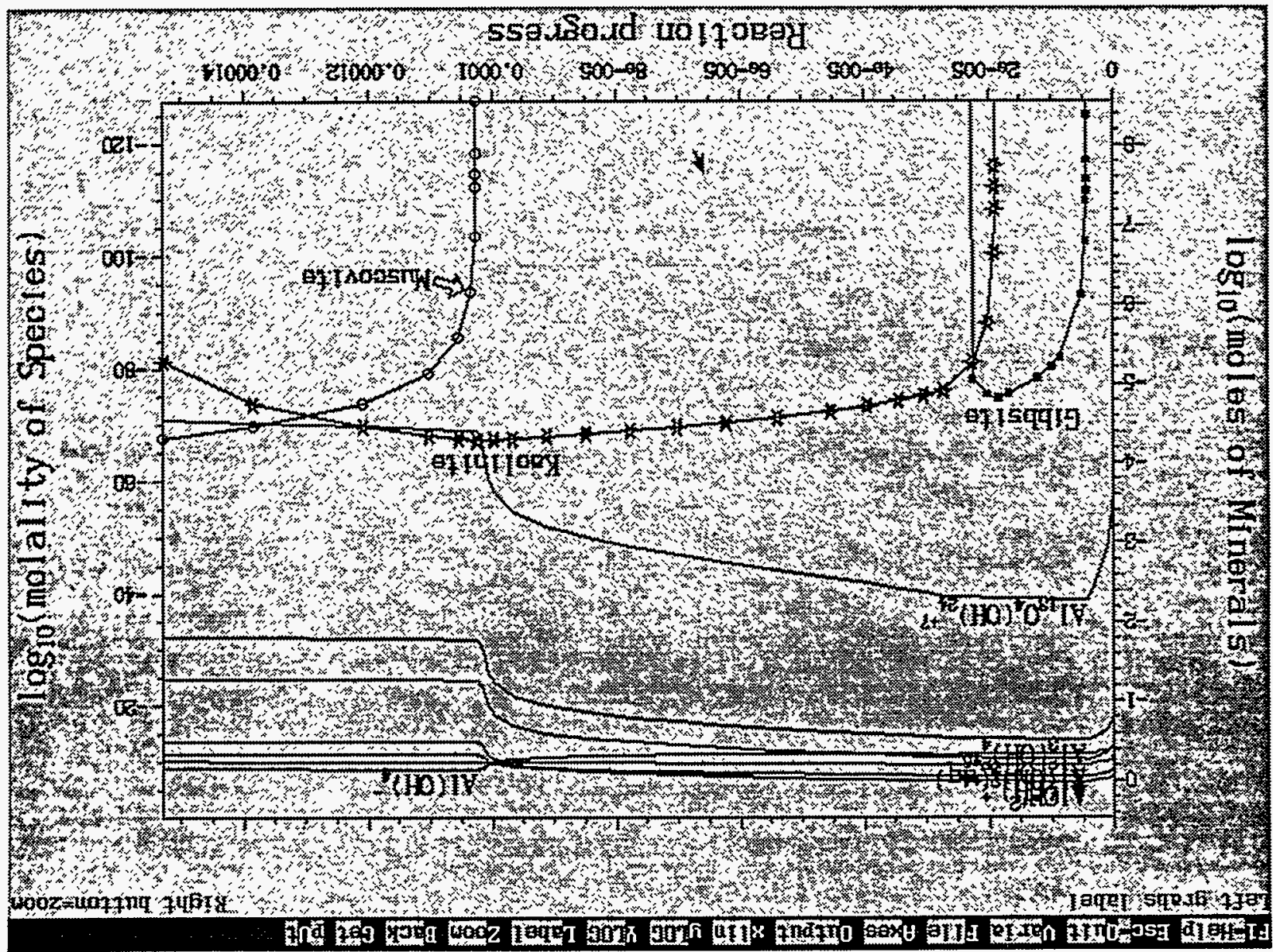




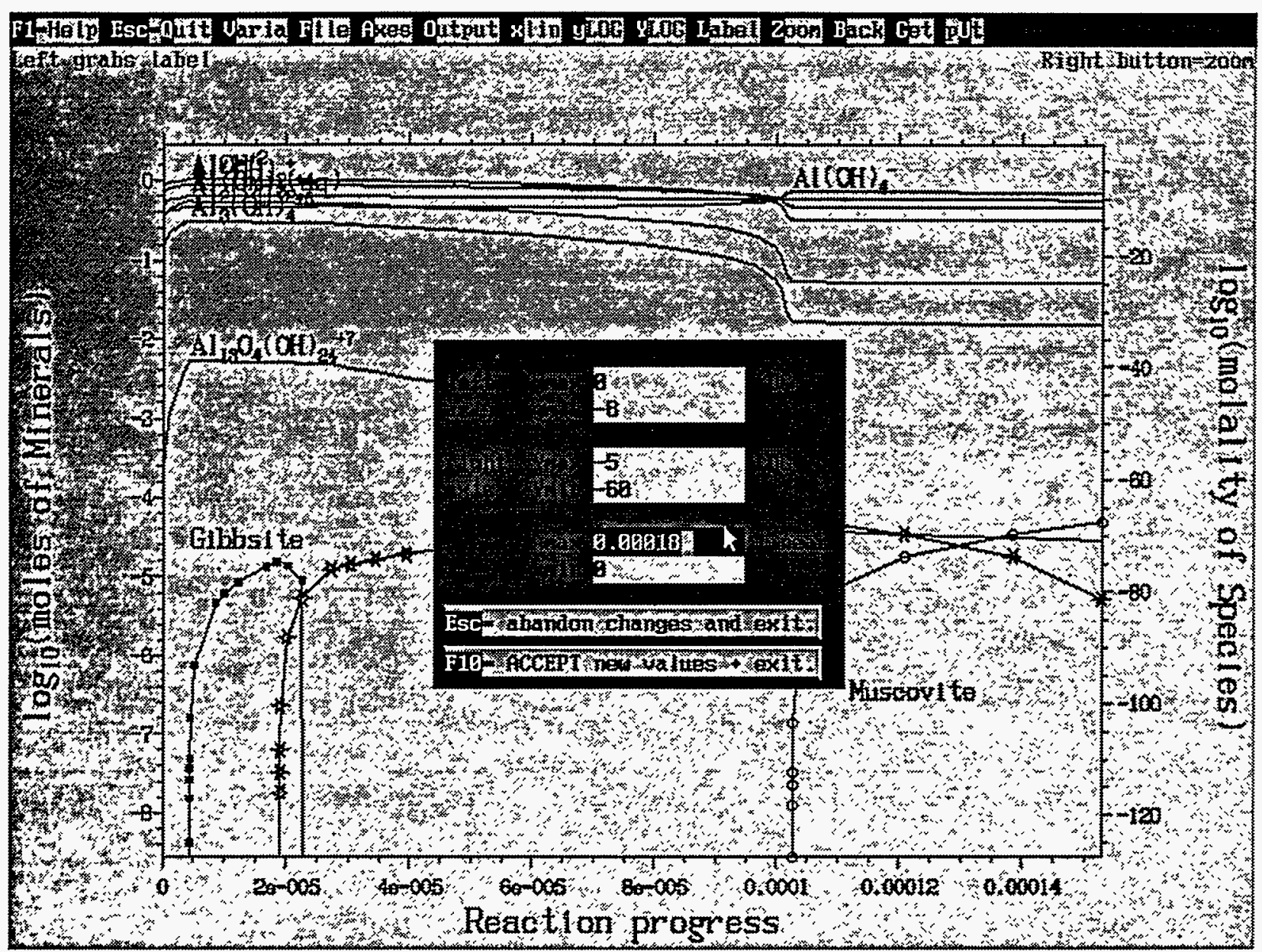

Figure 11. Setting axis ranges with the Axes dialog.

Click the mouse on the fires button (or press ' $\mathrm{A}$ ' on the keyboard). Edit the entries as shown in figure 11: the left axis from -8 to 0 , the right from -60 to -5 , and the $\mathrm{x}$ axis from 0 to 0.00018 . Then click on F10 2 ACCEPT new values fexit? (or press the F10 on the keyboard). Delete the curve for $\mathrm{Al}_{13} \mathrm{O}_{4}(\mathrm{OH})_{24}{ }^{+7}$, since this species has low concentration.

Send the plot to a graphics file by clicking on the Ditput button (or press ' $O$ ' on the keyboard). The screen updates slowly as PP writes each HPGL instruction to a default file named hp.000 in the current directory. 


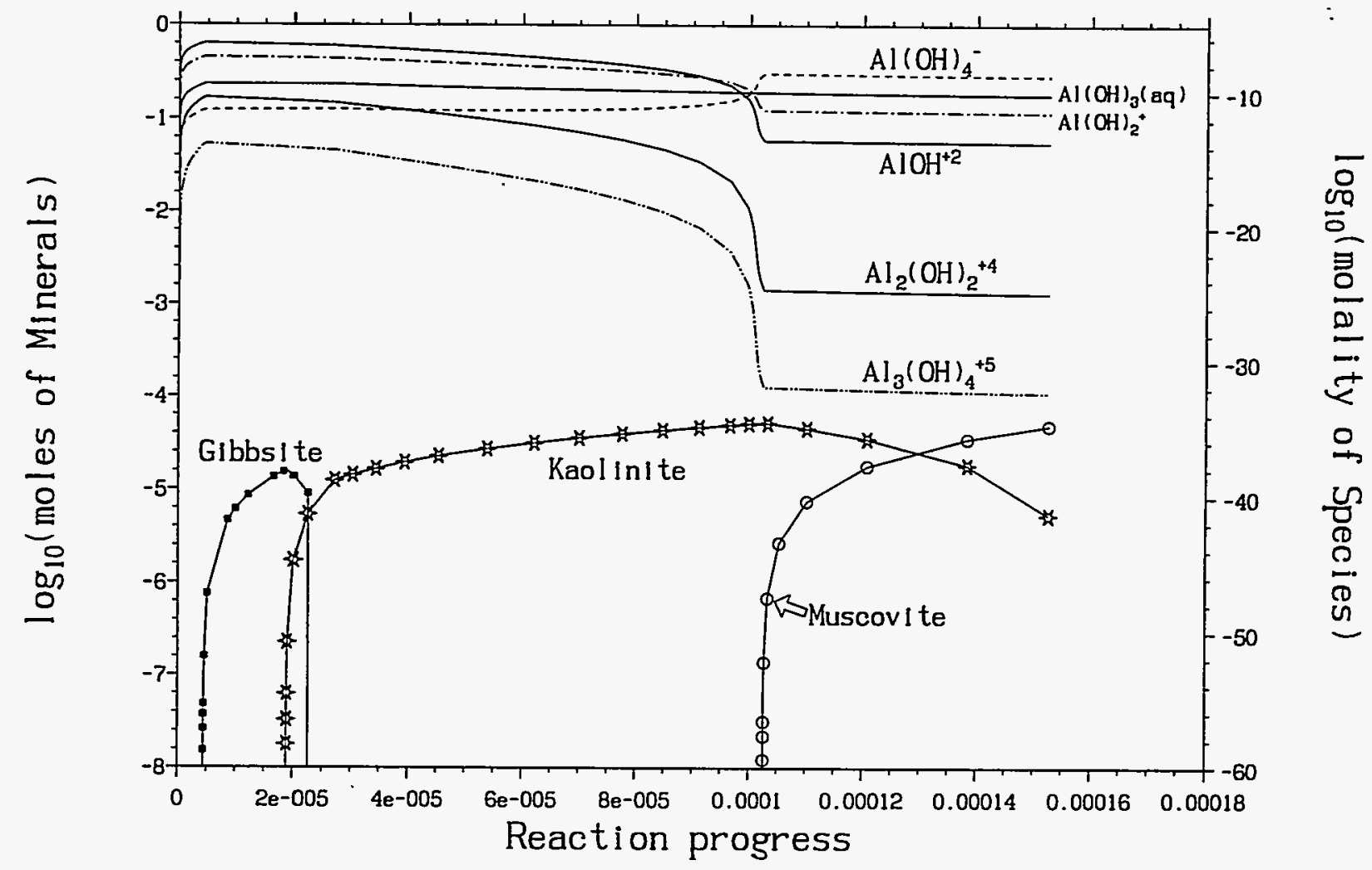

Figure 12. PP graphics file hp.000, imported into WordPerfect.

PP produces HPGL (Hewlett-Packard Graphics Language) graphics files. Section 5.13 describes how to import these files into several popular applications, and how to print them directly to HP III and 4 laser printers. Figure 12, for example, was imported directly into this report with the WordPerfect 5.2 "Graphics-Figure-Retrieve" dialog.

We now consider a more complex plot, to illustrate the processes of importing data, editing labels, and searching the EQ3/6 database. The next example requires successive runs with the EQ6 input files t4a.6i and $t \mathbf{4 b . 6 i}$ from the PP distribution. These input files were used to simulate the interaction of dissolving Calcite and Gypsum (run \#1) and pure Calcite (run \#2) with buried uranium oxide waste.

First create the hwsdata file by running EQ6:

runeq6 com t4a.6i

Then invoke PP, and click on the $\mathrm{pH}$ (in the top or "King" section), then click on the "ELEM" s icon (or press F2) to mark these variables for export. Export the data by clicking on Export (or by typing 'E' on the keyboard). Press "Enter" ( $\hookleftarrow$ ) on the keyboard to accept the default txt.000 filename. 


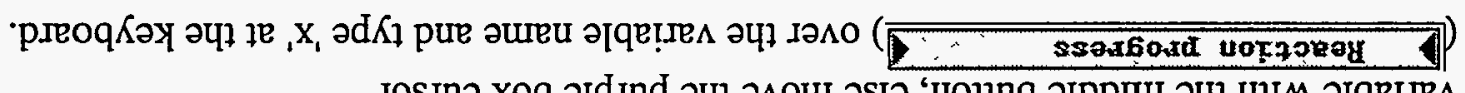

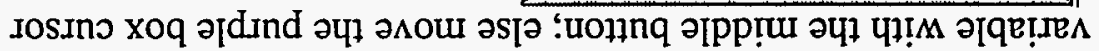

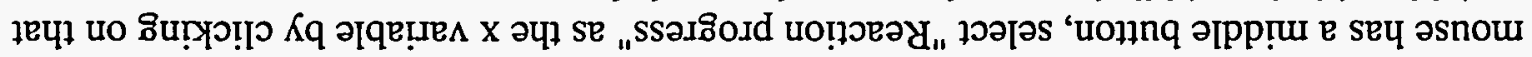

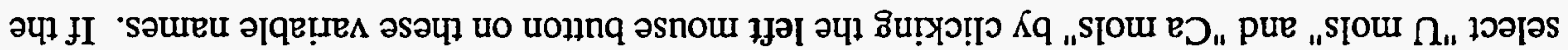

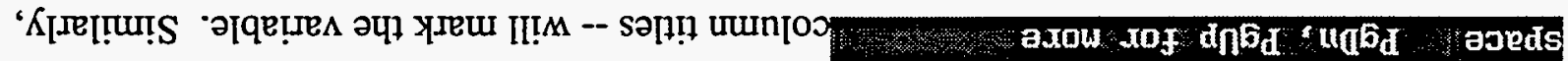

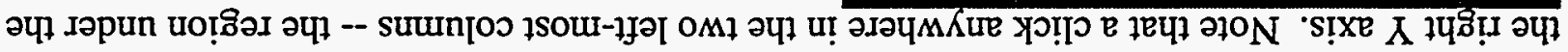

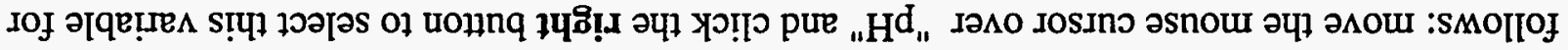

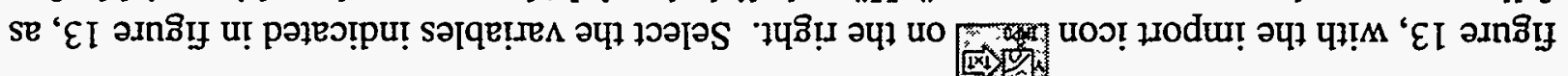

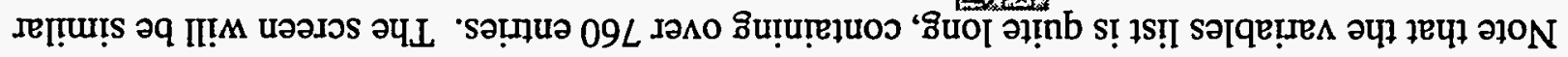

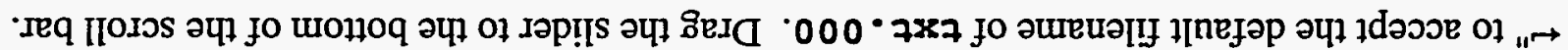

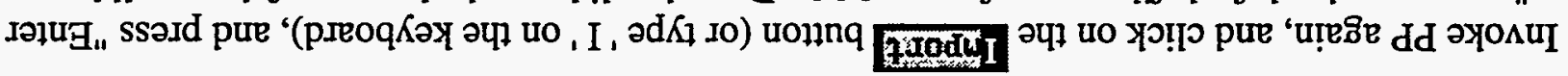

$\rightarrow$ T9・977 mos 9bounx

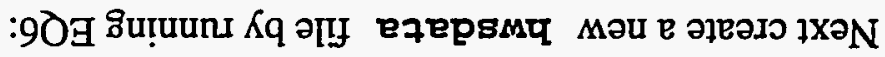

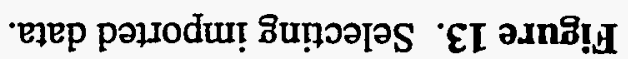

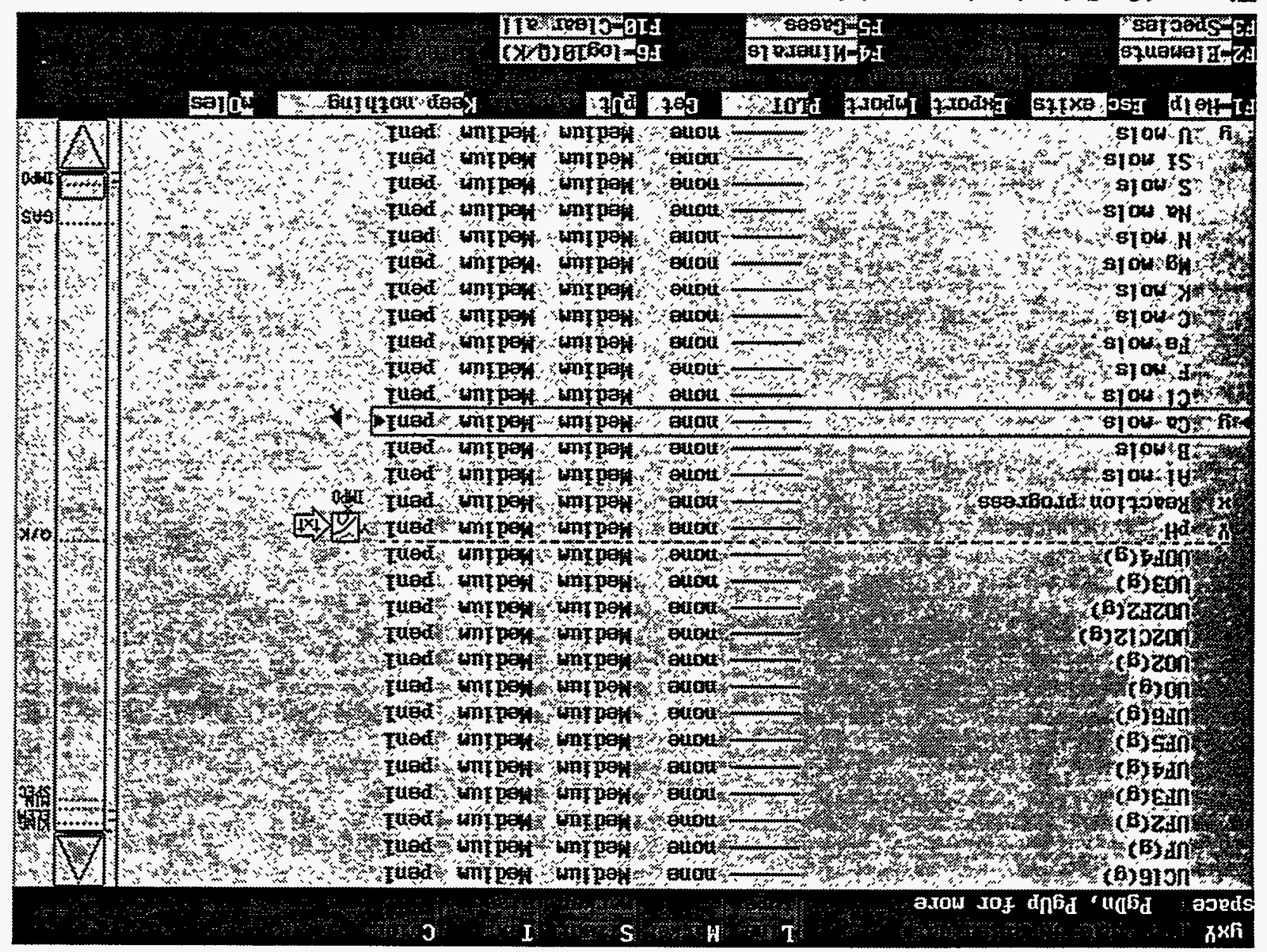




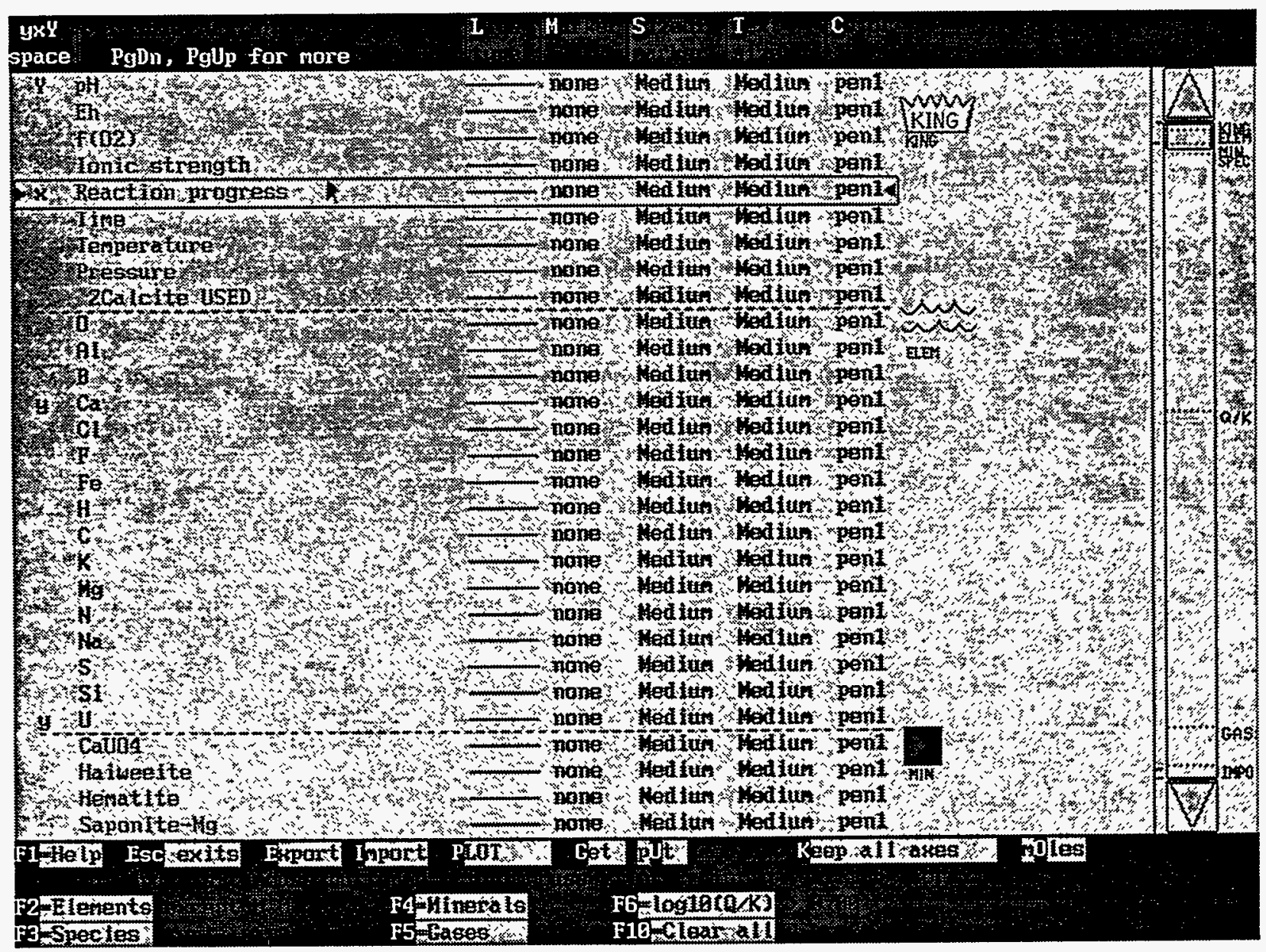

Figure 14. Selecting variables at the top of the list.

Next move the slider on the scroll bar to the top of the variables list and select the analogous variables, as shown in figure 14. Click on the PLOT 


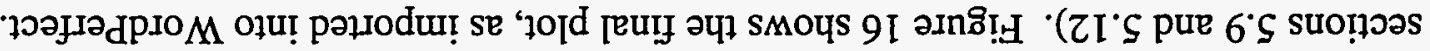

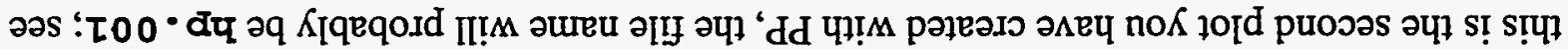

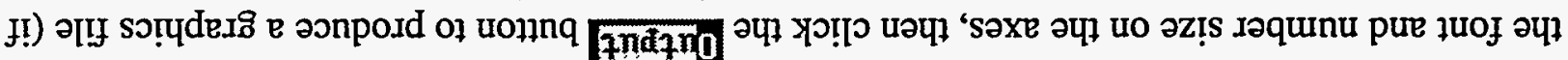

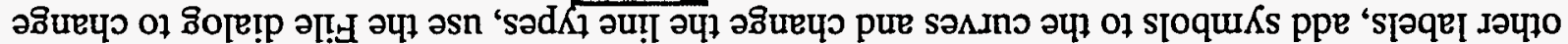

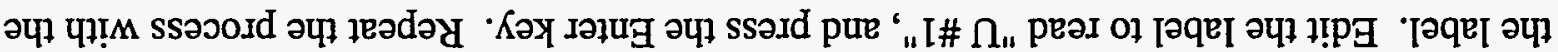

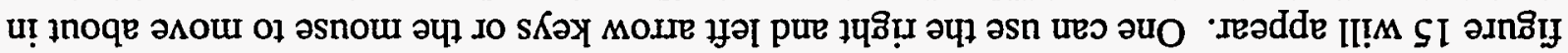

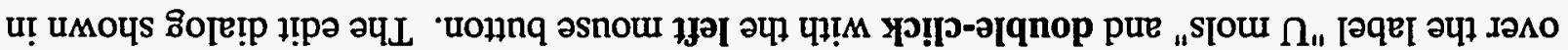

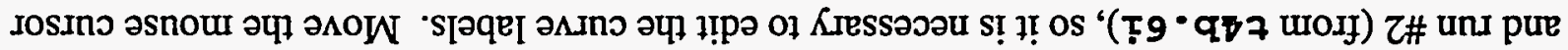

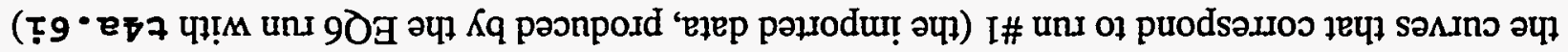

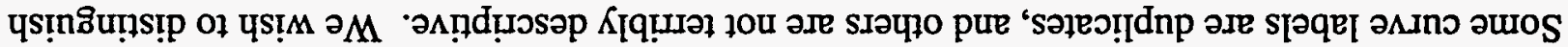

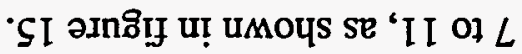

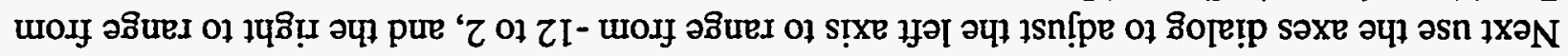

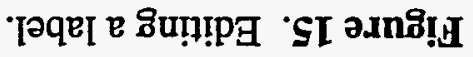

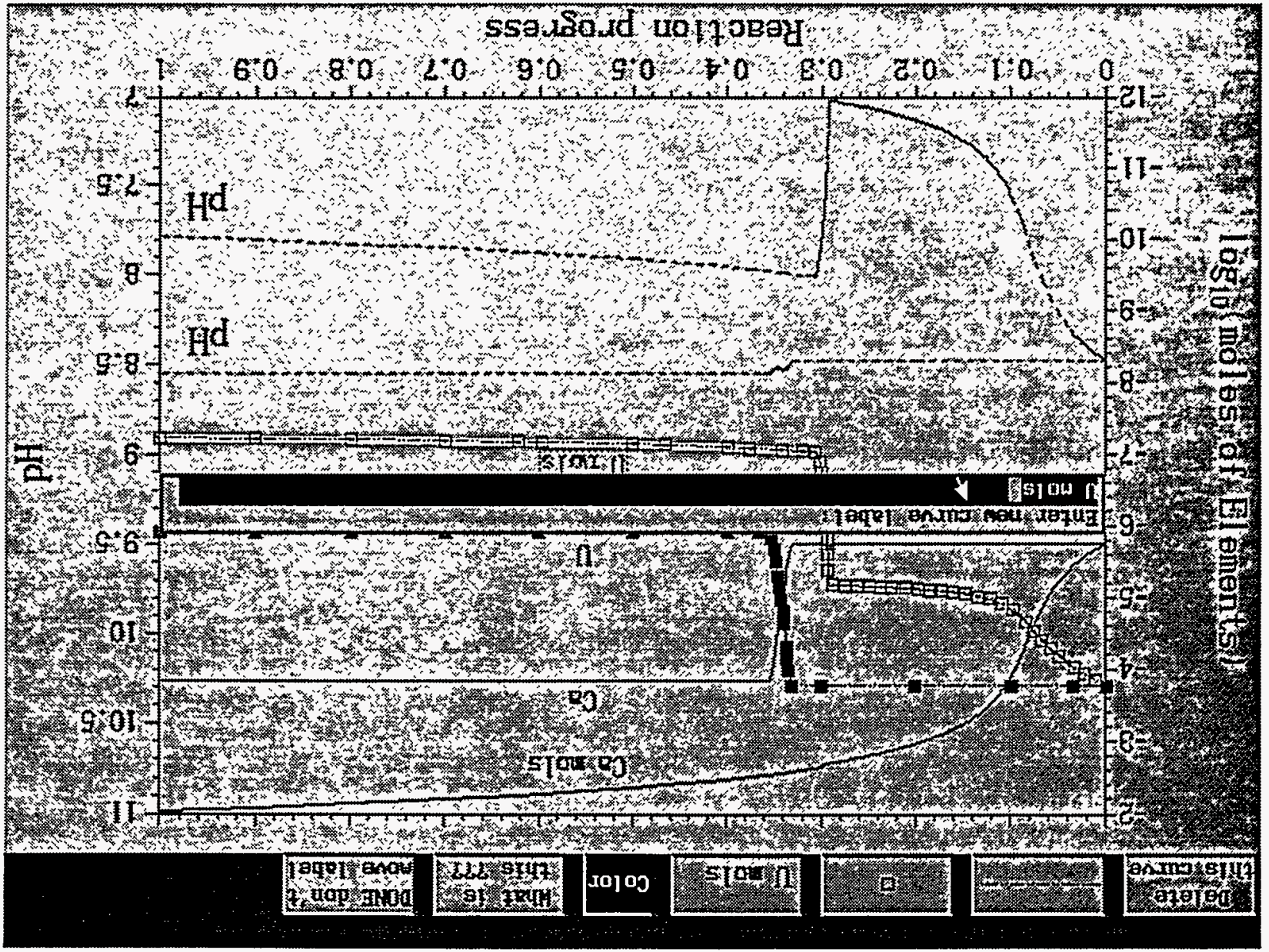




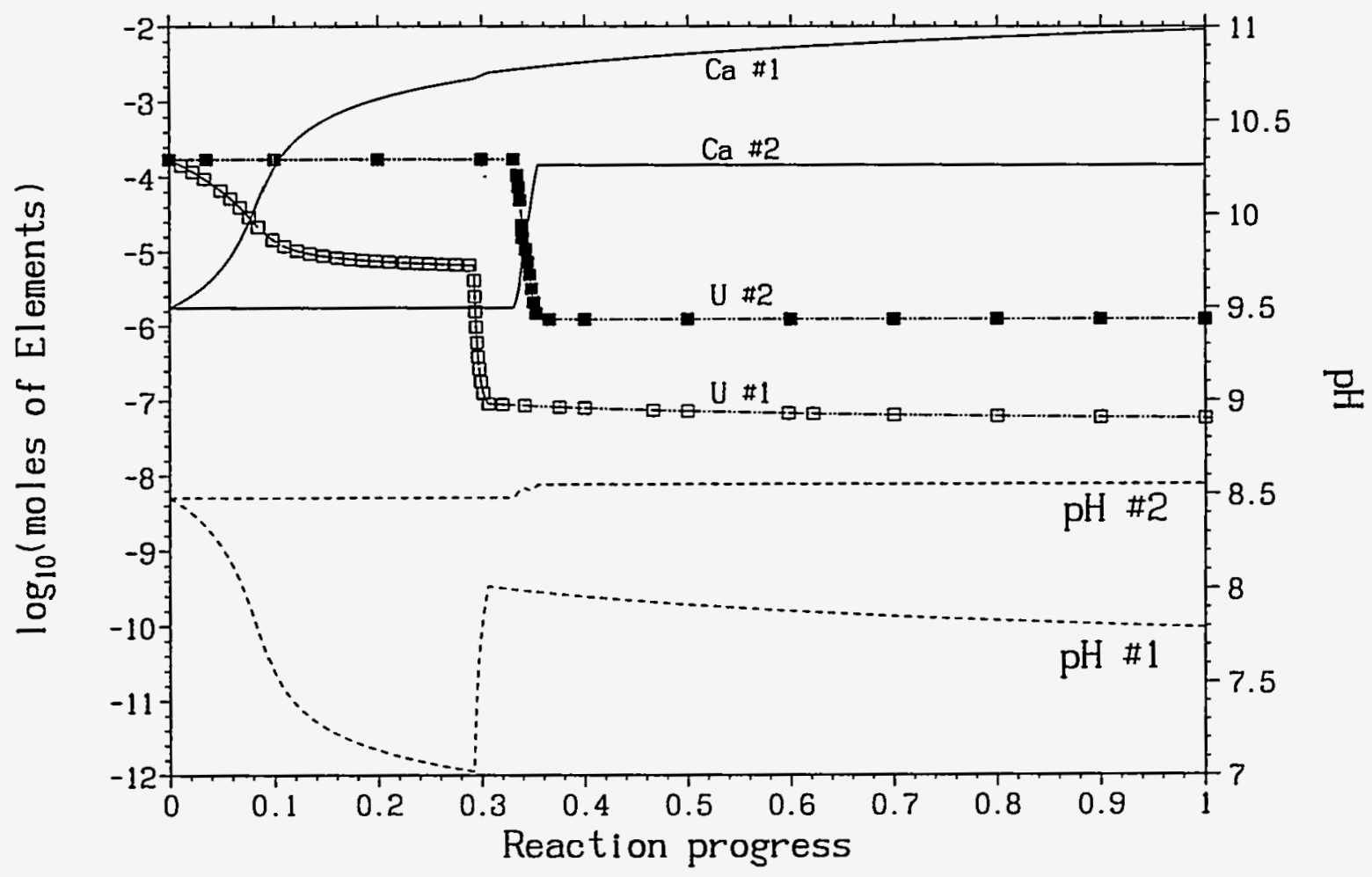

Figure 16. Graphics file from figure 15, imported into WordPerfect.

Next we examine PP's ability to search the EQ3/6 database. Click on Uaifia to return to the variables menu, and drag the scroll bar slider about $1 \mathrm{~cm}$ below the red " $\mathrm{Q} / \mathrm{K}$ " label to the right of the scroll bar, as shown on the right side of figure 17. Note the large number of Clinoptilolite entries; move the purple bar cursor over "Clinoptilolite-Ca" and type either '/' or '?' on the keyboard. The database search window will appear in the center of the screen with the corresponding entry in the EQ3/6 database. Typing ' $R$ ' at the keyboard will send the entry to both the printer and an appended file thermo.txt. Typing 'I' will switch to the "In CONTENTS" mode, which shows a compressed list of all the minerals and species in the EQ3/6 database used for the calculation, as shown in figure 18. One can scroll through these lists, examining data and even the references to the data, flipping between the "Text" and CONTENTS modes with the Enter key. 


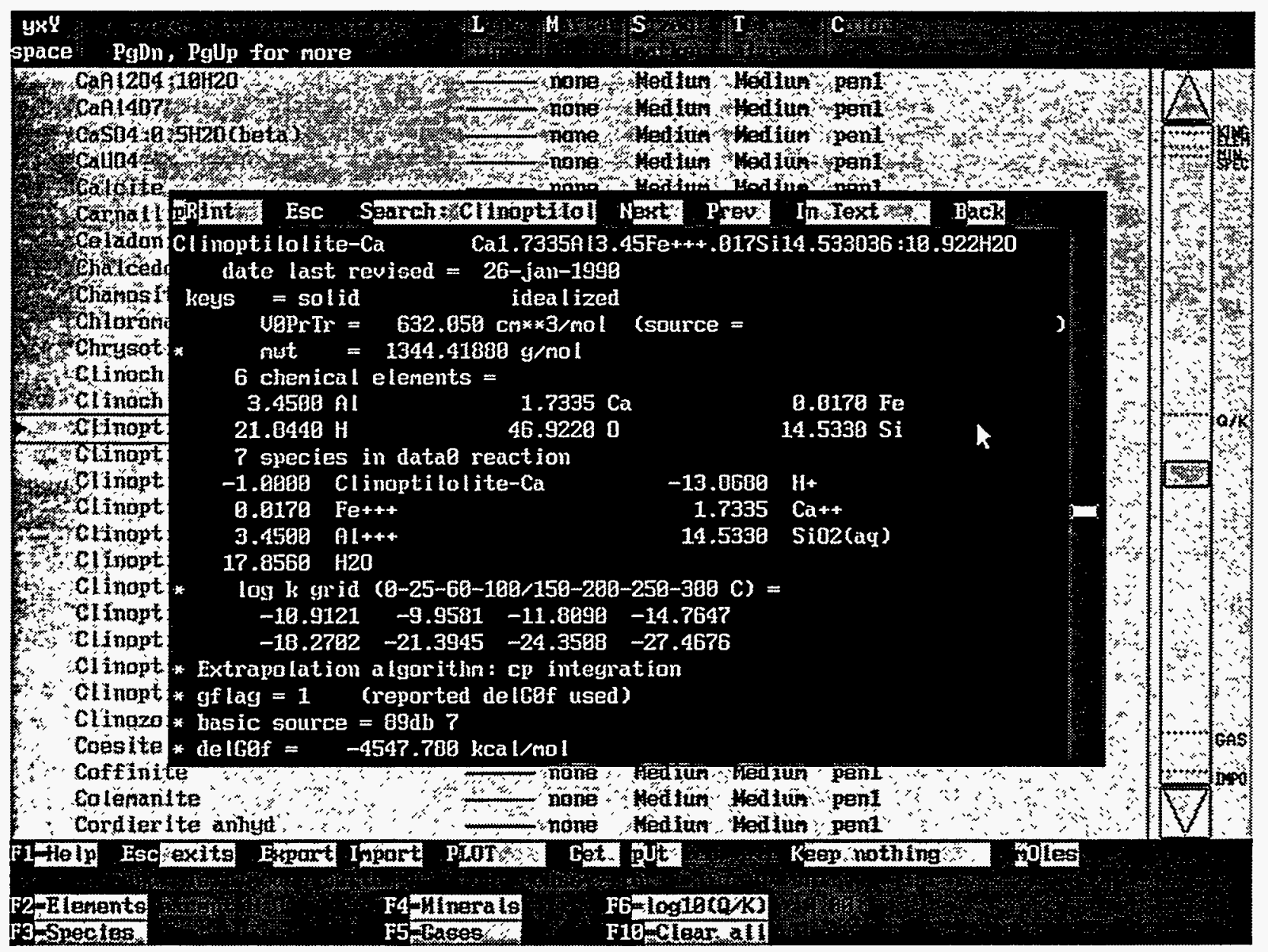

Figure 17. Searching the EQ3/6 database. 


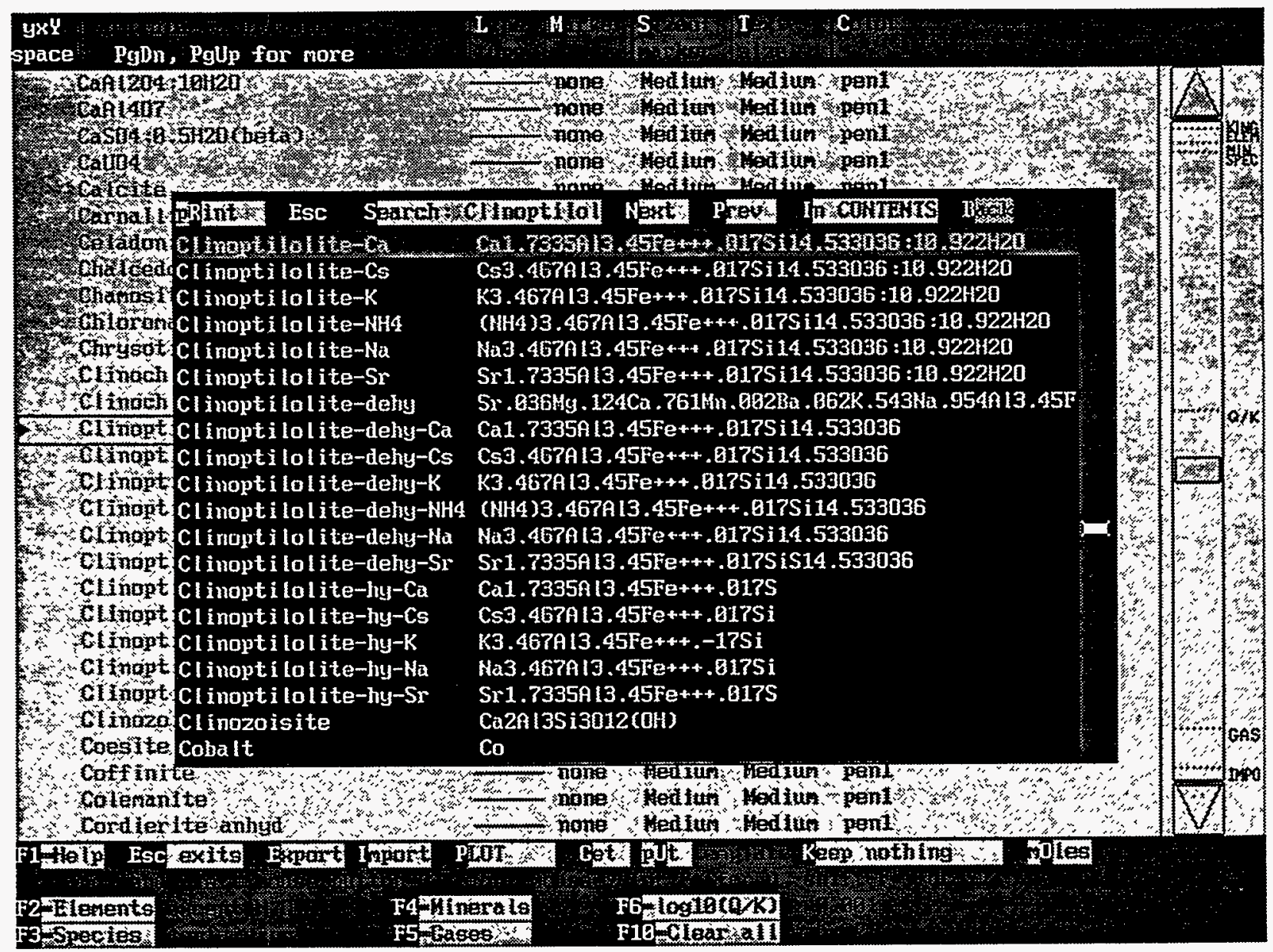

Figure 18. CONTENTS mode for searching the EQ3/6 database.

Note that the EQ3/6 database can also be searched from the plot screen. To do so, left-click on the label of interest, move the mouse cursor onto the this s? again.

The following four sections explain how to obtain help on-line, discuss the operations performed in the tutorial in greater detail, and explain how one edits the fonts and creates custom characters like those shown in figure 22. 


\section{Obtaining Help On-Line}

Help is obtained by pressing the F1 key in the variables screen, in the plot screen, and in several sub-menus. The help window (figure 19) has three parts: 1) the main window, with a black background; 2) the Scrollbar on the right side, with a white slider that shows the current position in the help file; and 3) the menu bar at the top.

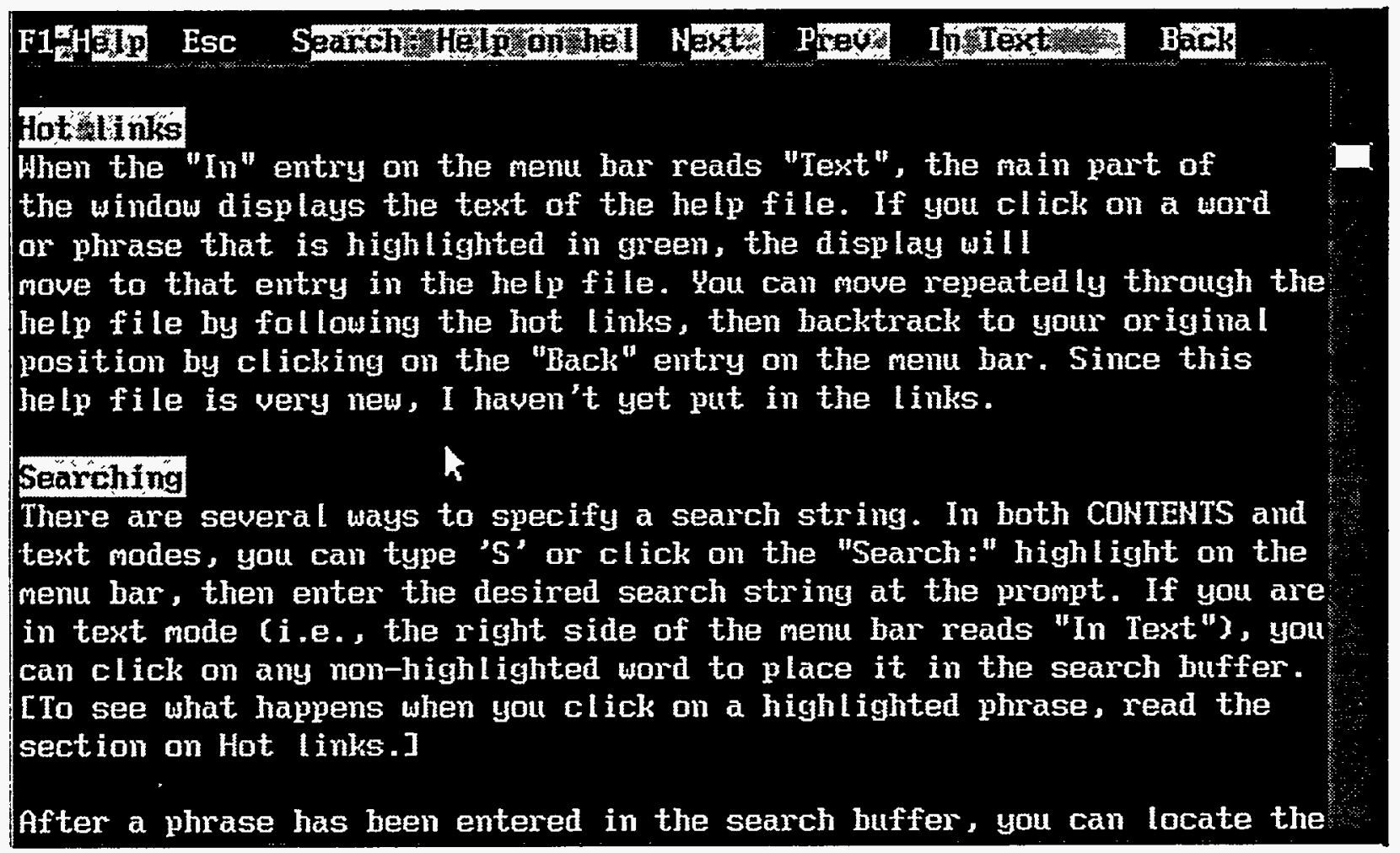

Figure 19. The help screen.

The main window displays either the text of the help file, or its table of contents. One can navigate through either by using the PgUp, PgDn and cursor keys. Alternatively, one can use the mouse to drag the slider on the scrollbar. To see the table of contents, type ' I', or click on "In Text".

The menu bar has 7 selections:

\begin{tabular}{|c|c|}
\hline $1=$ Help & - explains how to use the on-line help; \\
\hline Esc & exits help; \\
\hline arch: & - allows one to enter search text for the "Next" and "Prev" commands; \\
\hline 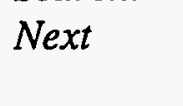 & $\begin{array}{l}\text {-- searches forward through the help file to find the next occurrence of the text } \\
\text { entered via the "search" command; }\end{array}$ \\
\hline Prev & -- searches backward to find the previous occurrence of the text; \\
\hline $\ln \ldots$ & $\begin{array}{l}\text {-- indicates whether the main display is showing the CONTENTS, or the text } \\
\text { of help file -- typing ' I ' or clicking in the highlight bar toggles the mode; }\end{array}$ \\
\hline & allows one to retrace steps, after using hot links to travel through the file. \\
\hline
\end{tabular}


One can choose a selection by clicking the left mouse button on the appropriate portion of the menu bar, or by typing the highlighted letter or key -- for example, typing 'S' brings up the search string editor.

When the "In" entry on the menu bar reads "CONTENTS", the help window displays a list of the main topics in the help file. There is a pink and green highlight bar across the screen, which can be moved up and down with the cursor keys or the mouse. Clicking on a contents selection with the mouse, or pressing the Enter key, moves to that entry in the help file.

\subsection{Hot Links}

When the "In" entry on the menu bar reads "Text", the main part of the window displays the text of the help file. Clicking the mouse pointer on a word or phrase that is highlighted in green causes the display to move to the associated topic in the help file. One can move repeatedly through the help file by following the hot links, then backtrack to the original position by clicking on the "Back" entry on the menu bar.

\subsection{Searching}

There are several ways to specify a search string. In both CONTENTS and text modes, one can type ' $S$ ' or click on the "Search:" highlight on the menu bar, then enter the desired search string at the prompt. In text mode (i.e., the right side of the menu bar reads "In Text"), one can click on any non-highlighted word to place it in the search buffer.

After a phrase has been entered in the search buffer, locate the next occurrence of that phrase by typing ' $\mathrm{N}$ ' or ' + ', or by clicking on the "Next" highlight bar. Similarly, find the previous occurrence in the file by typing 'P' or '-' or clicking on "Prev".

\subsection{Editing the Help File}

The help file, help_pp, is mostly ASCII with a few pairs of formatting characters. To edit this file, use an ASCII editor such as DOS edit or the Windows notepad, and set the word-wrap on the 70th column. Unless one adds Hot Links or titles, it will generally not be necessary to add the formatting characters. The formatting characters consist of either ASCII 28 or ASCII 249 (PP regards the two as equivalent), followed by one of the letters u,U,d,D (for "up" and "down", used to sub or superscript text), $\mathrm{x}, \mathrm{X}$ (to turn on xor mode), $\mathrm{n}, \mathrm{N}$ (to turn off xor) or $\mathrm{w}, \mathrm{W}, \mathrm{b}, \mathrm{B}, \mathrm{c}, \mathrm{C}$, $\mathrm{g}, \mathrm{G}, \mathrm{m}, \mathrm{M}, \mathrm{y}, \mathrm{Y}$ to turn on a particular color and brightness (white, blue, cyan, green, magenta, yellow, where capital letters denote bright colors). In DOS edit, ASCII 28 appears as a midline dot $(\bullet)$, and in notepad it may appear as and accented $\mathrm{u}(\mathrm{u})$, depending on the active font. Both editors allow these characters to be replicated with the copy and paste commands.

There is one minor caution about editing help_pp. The help utility is entered through the $\mathrm{C}$ function help (char *strng), which is mildly context sensitive, and finds its location in the help file be searching for the target "strng". Typically, "strng" refers to part of a section title in help_pp. Thus if section titles are arbitrarily changed, help () may not locate the appropriate section, and will simply default to the top of the help file. 


\section{The Variables Screen}

The variables screen is divided into three horizontal regions, with pink (top of screen), white (middle), and dark cyan (bottom) backgrounds (figure 1).

The top (pink background) region lists what is in the columns of the middle region. The highlighted hot keys can be used to toggle through the choices in the columns. It is generally much easier to use the mouse to change the choices; just move the mouse cursor to the column and row to be changed, and click on the entry repeatedly to toggle through the choices.

The middle region displays the variables that can be plotted, along with their plotting attributes. The right side shows a scroll bar that represents the entire list of variables. The divisions between types of variables are marked on the scroll bar with dotted lines, and to the right there are abbreviations (e.g. "SPEC" for species) to indicate the types. The left and main part of this region is a "window" into the list of variables. The size of the window corresponds to the height of the slider (the slider is the light blue-green rectangle on the scroll bar). The scroll bar is the fastest way to navigate through the variables list; move the mouse pointer over the slider, then depress the left mouse button and hold it down while moving the slider to a new position. When the button is released, the left part of the screen will show the variables in this new region.

When the mouse pointer moves across the white region, a purple cursor bar follows along. The cursor bar can also be moved with the cursor keys (arrow keys), PgUp, and PgDn. We call the variable included in the cursor bar the "current" variable.

\subsection{Selecting Variables (Variables Screen)}

One can select variables to plot with the keyboard or mouse. Typing $x, y$, or $Y$ causes the current variable to be selected as the horizontal, left vertical or right vertical axis, respectively. One can select any number of $\mathrm{y}$ or $\mathrm{Y}$ variables, but only one $\mathrm{x}$ variable for the original (nonimported) data, and one $\mathrm{x}$ for the block of imported data. Pressing the space bar, backspace or delete key erases the $\mathrm{x}, \mathrm{y}$ or $\mathrm{Y}$ on the current variable.

To select variables with the mouse, move the mouse cursor over the variable name, then click the left button to select the variable as y (plots on left vertical axis), or the right button to select it as $\mathrm{Y}$ (right axis). If the mouse has a middle (third) button, clicking the middle button selects the current variable as $\mathrm{x}$. A second click (with the same button) erases an entry. Note that as variables are selected, they are also marked as ticks to the left of the scroll bar, so it is possible to see a summary of all the selected data, even when the variables list contains tens of pages.

\subsection{Plotting Attributes (Variables Screen)}

The columns to the right of the variable name give the plotting attributes: the Line type used to connect the points on the plot, the type of Marker (marker=symbol, such as square or circle) plotted at each point, the Size of the marker, the size of the Text used for each curve label, and the Color ("pen") used to plot the lines, markers and labels. To change an attribute for a particular curve, move the mouse cursor over the column and row of the attribute, and click the left mouse button repeatedly to step through the choices. To make changes with the keyboard, 
use the UP and DOWN arrow keys to position the cursor bar over the desired variable, then type the hot key (letter) corresponding to the column to be changed. The hot keys are shown at the top of each column, and are highlighted in bright white on a dark green background:

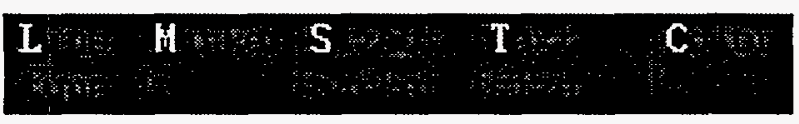

For example, type ' $\mathrm{L}$ ' repeatedly to toggle through the line type choices for that variable. All these attributes can be edited in the plot screen as well (perhaps much more intuitively), so it is not necessary to get all the selections exact.

\subsection{Icons and Variable Groups (Variables Screen)}

To the right of the attributes are some simple icons, denoting the following groups of variables:

KING -- a yellow crown, denotes what are sometimes called "master" variables, such as $\mathrm{pH}, \mathrm{Eh}$, reaction progress and time;

ELEM -- blue waves, denotes the bulk elemental composition of the aqueous phase in moles;

MIN -- a reddish octahedron, denotes the amounts of minerals present in the system, in moles;

SPEC - - waves with + and - signs dispersed throughout, denotes the molalities of dissolved species in the aqueous phase;

$Q / K \quad--$ a ghosted ("dissolving") octahedron, denoting the saturation state of all possible minerals or solid phases;

GAS -- a cloud to denote gas fugacities;

IMPO -- a distinctive icon, present only when data are imported.

The icons are placed at the top of each group of variables. The variable groups are further distinguished by alternating gray or black text for the variable names, and dashed line separators at the start of each group.

Clicking on the ELEM, MIN, Q/K, GAS or IMPO icons selects some subset for plotting. Clicking with the left button selects the variables for the left axis (y); the right button selects them for the right axis (Y). Clicking on the ELEM icon selects all elements but hydrogen and oxygen; clicking on the MIN icon selects all minerals; clicking on $\mathrm{Q} / \mathrm{K}$ selects all minerals with saturation states above 0.001 ; clicking on the IMPO icon selects the first imported variable as $\mathrm{x}$, and the rest as y or Y. Clicking on SPEC (species), however, brings up a search dialogue. All species names containing the entered substrings will be selected for plotting. One can select species based on more than one word fragment. For example, to find all species with both $\mathrm{Ca}$ 
and $\mathrm{O}$, one would type "Ca \& $\mathrm{O}$ " at the prompt (without the quotes).

\subsection{Buttons (Bottom of Variables Screen)}

The bottom of the variables screen contains light cyan "buttons" with red highlights:

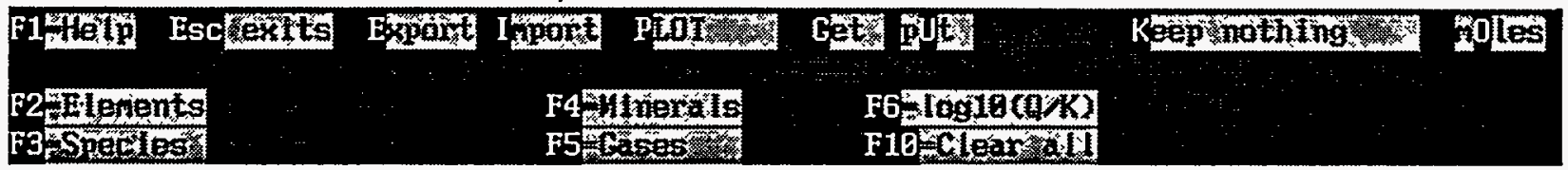

The button functions can be activated by clicking on the buttons with the mouse cursor, or by typing the red-and-white-highlighted key. The F1 button brings up the help screen (figure 19), and Esc exits the program. The F2 through F6 buttons mark subsets of the variables as indicated above, and duplicate the purposes of the icons, principally for use with lap-top computers. To mark y $v s$. Y subsets, one can click the left or right mouse buttons, respectively, or type the function key alone for $y$, and shift-function key to select $Y$. The F10 key clears (deselects) all the $\mathrm{y}$ variables, and shift-F10 clears all the $Y$ variables. Actions performed by the remaining buttons are slightly more complicated and are discussed in detail below.

\subsection{The PLOT Button and Sorting (Variables Screen)}

There are three ways to plot the selected data (and thus leave the variables screen). First, one can click on the PLOT bar at the bottom of the menu; second, just type 'p'. Third, typing 'control p' (that is, holding down the control key while pressing ' $p$ ') sorts the $x$ axis before plotting.

How is the 'control p' option different? Normally, PP plots the $\mathrm{x}, \mathrm{y}$ and $\mathrm{x}, \mathrm{Y}$ pairs in the order they were created by the reaction path program. Sometimes the result can be a little disconcerting; in a plot of aqueous composition vs. $\mathrm{pH}$ for example, the curves may wrap back on themselves, since $\mathrm{pH}$ may rise and fall in a calculation. By using the 'control $\mathrm{p}$ ' option, the $\mathrm{x}$ data will be sorted to avoid the wrap-back.

\subsection{Templates: Saving (pUt) and Retrieving (Get) Plotting Attributes (Variables Screen and Plot Screen)}

Often successive reaction path calculations use very similar conditions, and it is desirable to plot the results of each calculation with the same axis ranges, same symbols and line type, etc. The "pUt" command (in both the variables and plot screens) makes it possible to save templates that preserve the axis ranges, symbol types and sizes, font, text size, etc. Typing ' $U$ ' or clicking on the pUt button brings up a dialog box requesting a template name, with a default of template.tmp. The "Get" command (type 'G' or click on Get) performs the inverse, retrieving a template, again with the default name template.tmp.

The template files are ASCI, and can be edited, but do so at your own risk, since the order of data in the templates is important.

\subsection{Exporting Data (Variables Screen)}

The "Export" button at the bottom of the screen saves the data marked with $x, y$ or $Y$ to a tab-delimited ASCII (text) file. This type of file can be read, transparently, by many spreadsheet 
and graphics programs. In the ASCII file, each column will represent a variable. The first row of the column will be the variable name, and the following rows will be the values of the variable at successive steps in the calculation.

Clicking on Export (or typing ' $E$ ' at the keyboard) brings up a dialog box requesting a file name; pressing the return key or clicking outside the box accepts the default file name (initially txt.000). PP autoincrements export file names, so the next default name would be txt.001, etc.

\subsection{Importing Data (Variables Screen)}

The "Import" button performs the inverse of Export. Clicking on Import (or typing

' I') brings up a dialog box with the default file name txt.000. PP expects the import data to be in columns of equal length, and the first entry of each column is assumed to be the curve label (if the columns are of unequal length, pad them with repeats of the last real datum). Spaces, commas, and tabs may be used as delimiters, and MUST NOT appear in the labels or data entries. If it is necessary to indicate spaces in a label, use underscores, which PP will convert into spaces. Here is an example of a valid import file:

\begin{tabular}{lcc}
\multicolumn{3}{l}{ wombat_ears $\mathrm{pH}$ p } \\
0.05 & 1.0 & $1 \mathrm{e}-7$ \\
0.1 & 2.0 & $1.2 \mathrm{e}-7$ \\
.13 & 2.5 & $1.3 \mathrm{e}-7$ \\
.17 & 3.0 & $3 \mathrm{e}-7$ \\
.25 & 3.3 & $1 \mathrm{e}-6$ \\
.36 & 3.45 & $1 \mathrm{e}-5$ \\
.56 & 3.6 & $1 \mathrm{e}-4$ \\
.7 & 3.6 & $1 \mathrm{e}-4$ \\
.9 & 3.6 & $1 \mathrm{e}-4$ \\
.99 & 3.6 & $1 \mathrm{e}-4$
\end{tabular}

The formatting requirements are not very strict. The columns can have varied numbers of spaces separating entries, and do not need to be aligned. The labels need not have any connection to the variables in EQ6.

If the data are imported successfully, PP will place them at the end of the variables screen in a section called "IMPO", with a distinctive icon. In the example above, PP would replace the underscores with spaces, creating variable names "wombat ears" and "pickle \#".

The main purposes of the import function are:

(1) to allow results of successive reaction path runs to be merged and viewed together;

(2) to superpose experimental results or literature values on the plot.

The tutorial contains an example of case (1) (figures 13 - 16). 
The imported data are regarded as "guests", and cannot be plotted by themselves; at least one : "host" $\mathrm{x}, \mathrm{y}$ or $\mathrm{x}, \mathrm{Y}$ pair must be selected from the other (non-imported) data. One can select one $\mathrm{x}$ and many $y$ or $Y$ variables in the imported section. If no $\mathrm{x}$ variable is selected, the imported data will not appear on the plot. Remember that step sizes in reaction path calculations vary from run to run, so don't expect the nodes of the curves to line up with the results of the current run.

\subsection{The Keep Button (Variables Screen)}

Note the "Keep" selection in the bottom part of the variables screen. Each time the user moves between the variables and plot screens, PP tries to guess if it should keep (preserve) the plot axis ranges and the label positions, or automatically rescale to accommodate new data selected in the variables screen. In effect, PP tries to save all the labor expended zooming the plot or moving labels. The "keep" button displays the current state of affairs, as judged by PP.

In most cases the user can ignore the Keep button, and accept PP's decisions about rescaling. However, PP's choices can be overridden. Clicking on the Keep button toggles through the following choices:

(1) Keep nothing - the next plot will rescale all axes and reposition labels near the curve maxima;

(2) Keep left $y$ axis - the next plot will preserve the scaling on the left $y$ axis, but rescale $\mathrm{x}$ and $\mathrm{Y}$ (right vertical axis) and move labels ;

(3) Keep right $Y a x$. - the next plot will preserve the scaling on the right $Y$ axis, but rescale $\mathrm{x}$ and $\mathrm{y}$ (left vertical axis) and move labels;

(4) Keep $y+Y$ axes - the next plot will preserve the scaling on the left $y$ and right $Y$ axes, but rescale $\mathrm{x}$ and move labels;

(5) Keep $y+x$ axes - the next plot will preserve the scaling on the left $y$ and $x$ axes, and rescale the right $\mathrm{Y}$; label positions associated with the left axis will be preserved;

(6) Keep $Y+x$ axes - the next plot will preserve the scaling on the right $\mathrm{Y}$ and $\mathrm{x}$ axes, and rescale the left $y$; label positions associated with the right axis will be preserved;

(7) Keep all axes - the next plot will preserve the scaling on all axes, and preserve all label positions.

Note that subsequent actions in the variables screen may still override a manual Keep selection. Suppose, for example, the user selected "Reaction progress" as the $\mathrm{x}$ variable for the plot, then toggled "keep" to selection (5) above (Keep $y+x$ axes). If the user subsequently changed the $x$ variable from "Reaction progress" to " $\mathrm{pH}$ ", PP would downgrade the "keep" selection to (2) (Keep left y axis).

The "keep" option is a mixed blessing; a user returning to the variables menu probably plans to 
add another curve, which may be way off scale if the axes are not rescaled automatically. If an expected curve seems to have vanished, try toggling the Ylin/LOG and ylin/LOG buttons on the plot screen, or return to the variables menu and toggle "Keep" to "Keep nothing" to force a rescaling.

\subsection{The mOles / MOLAL Button (Variables Screen)}

This button affects only the ELEM data (bulk composition of the aqueous phase), and toggles the plotting units between total moles and molal.

By default, data in the ELEM section are given in moles. Since EQ3/6 defaults to $1 \mathrm{~kg}$. of $\mathrm{H}_{2} \mathrm{O}$ for calculations, these data will often correspond to molality. However, in reaction paths that involve evaporation, it may be more appropriate to plot the molality of each element dissolved in the aqueous phase. In the future, a "ppm" (parts per million) option may be added as well. 


\section{The Plot Screen}

Selecting "PLOT" at the bottom of the variables screen brings up the plot screen. The program tries to pick reasonable axis ranges, and attempts to pick pleasing subdivisions for tick marks. The curve labels (e.g., $\left.\mathrm{Al}_{2}(\mathrm{OH})_{2}{ }^{+4}\right)$ :are placed near the highest position on the curves, and at this stage may overlap. However, it is easy to adjust the default scaling and labels.

The top of the plot screen contains a row of 14 buttons:

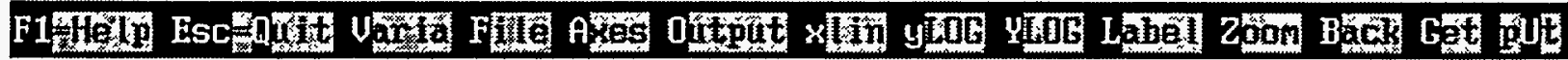

The buttons are activated by clicking on them with the mouse, or by pressing the key indicated by the red and white highlight. Below is a quick summary of the options available in the plot screen, as they are listed on the menu bar at the top of the screen:

F1 -- brings up the help screen;

Esc -- leaves the program (there is a chance to cancel);

Varia -- returns to the Variables screen;

File $\quad-$ brings up a menu for changing output file characteristics, including

-- output file name,

-- graphics format (pure HPGL $v s$. embedded printer codes),

-- font (default $v s$. Roman-like triplex),

-- use of Minor ticks,

-- size of numbers on axes,

-- pen width,

-- arrow size and type;

Axes -- brings up a dialog for changing axes ranges (see 5.2 "Zooming with the Mouse");

Output -- sends the plot to a file or hardcopy device ("prints" the plot);

$x, y, Y \quad--$ toggle the $\mathrm{x}, \mathrm{y}, \mathrm{Y}$ axes between LOG and linear display;

Label -- adjusts label positions by keyboard (see 5.3 Changing Curve labels);

Zoom -- zoom part of the plot by keyboard (see also 5.2 Zooming with the Mouse);

Back -- undoes the last zoom;

Get -- get (retrieve) plot Templates, i.e. stored axis ranges, variable selections; symbol (marker) and line types (same as variables screen Get);

pUt $\quad$-- put (save) a template to a file for later reuse (same as variables screen pUt). 


\subsection{Changing Axes Ranges and Scaling (Plot Screen)}

First off, the default choice of $\log v s$. linear scaling may be inappropriate. For example, $\mathrm{pH}$ should be plotted on a linear, rather than $\log _{10}$ scale. The defaults can be changed with the $x, y$, and $\mathrm{Y}$ toggles at the top of the screen, which affect the corresponding horizontal, left vertical and right vertical axes.

The axes ranges can be altered in several ways. If exact limits are desired, it is best to use the axes dialog (type 'A' or click on the "Axes" button at the screen top; figure 11), which allows one to type in the desired ranges. If exact ranges are not needed, it is often faster and more intuitive to zoom with the mouse.

\subsection{Zooming with the Mouse (Plot Screen)}

To zoom with the mouse (figures 7 and 8), move the mouse to one corner of the desired zoom area, click the right button, then move the mouse till the zoom box is the desired shape (if a mistake is made, type Esc to exit). Then click the right button once more to fix the shape of the zoom box. Clicking the right button once more INSIDE the zoom box rescales the plot to make the zoom box full screen. If one types Esc or clicks outside the box, the zoom will abort. To return to the previous scaling, click on the "Back" button on the top right side of the screen.

Note that before the final click is made inside the zoom box, there is an option to print the plot with the zoom area indicated as a dashed box. Just press the "O" key to create an output file with the dashed box, then click inside the zoom box to finish the zoom.

\subsection{Changing Curve Labels, Markers (Symbols) and Line Types (Plot Screen)}

The simplest way to move or edit the labels and change the curve symbols, curve color, line type, and label size is with the mouse (figures 3-5).

To edit a curve label, double-click on the label with the left mouse button. An edit dialog box will then pop up. Pressing the return key or clicking outside the box accepts the new label. To "double-click", press the button twice in rapid succession. The double-click delay is $\approx 0.35$ seconds, which is comfortable for most users.

To move a label or change the curve characteristics, single-click on the label with the left mouse button. The label is then "attached" to the mouse cursor, and can be moved to another position (it is NOT necessary to hold down the mouse button to "drag" the label). Typing ' $\mathrm{A}$ ' will draw an arrow to the nearest node on the curve; typing ' $E$ ' will erase an existing arrow (see section 5.8 for the method of changing the arrow type). Clicking another mouse button in the main part of the screen fixes the label in that new position. 
Single-clicking on a label places a new menu at the top of the screen, displaying seven buttons, six of which are light cyan (light blue-green):

\begin{tabular}{|c|c|c|c|c|c|c|}
\hline Dertetey & 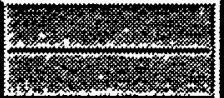 & ha synhol & goetand 12 & Color & 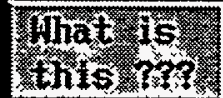 & Dove donyt \\
\hline
\end{tabular}

The left-most selection on the menu deletes the curve and label, providing a means to clean up a complex plot. The next button to the right allows one to change the line type used to connect the curves; clicking on this selection brings up a menu with available line types. Similarly, the next selection to the right allows one to change the size and type of symbol used on each plot point (figure 4). The next allows the user to select one of four text sizes for the label (figure 5), and the next selection makes it possible to change the color of the curve, symbols and text. The sixth button searches the EQ3/6 database for the thermodynamic and composition data of the label, so the user who has forgotten the composition of "Boehmite" can get a refresher (see section 1.2 "Installing PP" to make sure the search path is set correctly, and section 5.4 "Searching the EQ3/6 Database" for more detailed discussion). This menu reduces the need to return to the variables screen.

\subsection{Searching the EQ3/6 Database (Plot Screen and Variables Screen)}

From both the variables screen and the plot screen, one can obtain composition and thermodynamic information about the minerals and species in the variables list. In the variables menu, move the cursor over the variable of interest, then press either '/' or '?'. A screen similar to the help screen will come up with the corresponding entry in the EQ3/6 database (figures 17-18). There may be a delay of a second or so -- the databases are often several megabytes in size. One can then search through the database for any other species of interest; pressing the ' I' key toggles to a listing of all the available species data used by EQ3/6. If PP cannot find the database, it will display an error message; in such case, be sure the environment variables set correctly (see section 1.2, "Installing PP").

To search the database from the plot screen, right-click the mouse on the label of interest, then either choose the "What is this ???" button on the top right side of the screen, or type '/" or '?' at the keyboard (see also section 5.3 "Changing Curve Labels").

\subsection{Changing Axis Labels (Plot Screen)}

To change the axes (axis) labels, click over the label with the left mouse button. A dialog box will pop up with the current name. Pressing "Enter" or clicking outside the box uses the new value.

\subsection{Templates (Get and pUt) (Plot Screen)}

Templates can be saved and retrieved from the plot screen. See the discussion of Templates in section 4.6. 


\subsection{The Zoom and Label Buttons (Plot Screen)}

The "Zoom" and "Label" selections on the menu bar provide for keyboard-only editing on lap-top computers that lack a mouse or other useful pointing device. Each option contains two extensive submenus to describe each process. The mouse zoom and label operations are generally much simpler and allow more editing options. However, the keyboard-only process for moving labels has one distinct advantage; the curves and labels are selected by scrolling through the list with the up and down arrow keys, eliminating the need to "hunt and peck" for labels in a complex plot.

\subsection{File Options: Output File Characteristics, Plot Appearance (Plot Screen)}

Clicking on the "File" button (or typing 'F') in the plot screen brings up a menu with the following options (figure 6):

$$
\begin{aligned}
& \text { Esc -- clicking on this button leaves the File menu; } \\
& \text { output Filename }=\quad-- \text { allows one to change/edit the output filename; } \\
& \text { Graphics format }=\quad \text {-- toggles among "HPGL+laserjet codes", } \\
& \text { "pure HPGL (MS Word)", "HPGL WpWin 5.1/5.2"; } \\
& \text { Text font }=\quad \text {-- toggles between "Internal (simple)" and "External (Triplex)"; } \\
& \text { use Minor ticks = } \quad \text {-- toggles between "Yes" and "No"; } \\
& \text { number Size = -- toggles among small, Medium, *BIG* and HUGE! for the size } \\
& \text { of the numbers on the } x, y \text { and } Y \text { axes; } \\
& \text { pen Width }(\mathrm{mm})=\quad \text {-- selects the width of the plotter "pen" for HPGL files (affects } \\
& \text { hardcopy, not screen appearance); } \\
& \text { Arrow type }=\quad-\text { toggles among thin lines (no arrow head), and thin, Medium and } \\
& \text { FAT arrows with heads (figure 4); } \\
& \text { print } Q A \text { banner }=\quad-- \text { toggles between "NO" and "Yes"; the latter places a distinctive } \\
& \text { banner in the upper left corner of plots, to aid traceability. }
\end{aligned}
$$

The significance of several of these options is discussed in detail below.

\subsection{Changing the Output File Name (Plot Screen, File Submenu)}

The default output file names are $\mathrm{hp} .000, \mathrm{hp} .001, \mathrm{hp} .002$, etc. The name can be changed with the "File" option on the main menu bar. PP automatically increments most file names, starting with the extension. Thus if one chooses a file name of myfile.000, the first "Output" file will indeed be called myfile.000, but the subsequent times one clicks on "Output", the files will be named myfile.001, myfile.002, etc. If one were to chose a file name of dog, subsequent names would be doh, doi, doj, ... dpa, dpb, ... etc. See section 5.12 for information on how PP preserves file names between sessions.

If file the name is prn, 1pt1, or com1 to com4, the file will be sent to the hardcopy device (printer or plotter) on that port. If the file is sent directly to a hardcopy device, be sure the device is in HPGL mode, or choose "HPGL+laserjet codes" format in the File Options menu. 


\subsection{Changing the Text Font (Plot Screen, File Submenu)}

PP has two fonts -- a simple "internal" font, and a more complex, roman-like "external" or "triplex" font. To change the font, go to the Plot screen and type 'F' or click on 'File' (File Options) to bring up the submenu for graphics file options. Then type 'T' or click on "Text font" to toggle between the two choices: The external (triplex) font may look better, but it can make the HPGL file 3 times larger, and takes longer to print.

To use the external font, PP must be able to find the file external . fnt. To make sure PP finds this file, either place it in a directory called $\backslash \mathrm{pp}$, or the current working directory, or set the $\mathrm{PP}$ environment variable to point to the directory where the font is stored.

The internal font is compiled into pp.exe, and can not be changed. However, given patience it is possible to create a new external font with the program ff . exe. The later program can also be used to create custom symbols (markers), and is described in section 6 .

\subsection{The QA (Quality Assurance) Banner (Plot Screen, File Submenu)}

Selecting "Yes" places a banner in the upper left corner of the plot, e.g.:

$$
P W D=C: \backslash P P \quad O F=h p .004 \quad D B=\text { data0. com. R22a Machine ID=1528 08-17-94 12:12 }
$$
IEQ6 input file name $=\mathrm{mlcro} .61 \mathrm{BIN}=$ hwsdata $\mathrm{PPVER}=1.01$

The first line of the banner contains 6 entries. "PWD=" gives the present working directory, where $\mathrm{PP}$ was invoked. "OF=" is the name of the output file. " $\mathrm{DB}=$ " gives the name of the database used in the EQ3/6 run, along with its version number. "MachineID" gives the sum of the first 4096 bytes of the PC BIOS at $0 x f e 000$ (each byte is converted to an unsigned 32-bit integer before summing). Since machine BIOSes usually have dates and serial numbers, it is extremely likely that this number will uniquely identify the machine used for the plot. The last two entries on the first line give the date and time of the plot.

The second line of the banner has three items. The first item is the first line of the EQ6 input file (not including the "l---.-" line for the EQ6 "D" format). It is customary to place the input file name on the first line of an EQ6 input file, providing traceability. The second item, "BIN=" is the name of the binary plot file read by PP. The third, "PPVER=" gives the PP version number.

\subsection{The Preferences File (prefer.pp)}

When PP terminates, it saves all the selections in the File Submenu to a binary file called prefer.pp. The next time PP is invoked, it searches the working directory for this file. If prefer.pp is found, PP reads in the preferences, including the next name for the graphics output file. Thus if one prefers to always use the triplex font, one should not have to go to the File menu every time $\mathrm{PP}$ is invoked to make this selection. This process takes place transparently. Since PP only searches the local directory for prefer.pp, a separate copy can be kept in each subdirectory, reflecting different preferences for different sets of reaction path 
runs. Deleting this file returns the File Submenu to its defaults.

Note that the "next" graphics output filename is stored in prefer.pp. Thus if one invokes PP and saves files $\mathrm{hp.000}$ and $\mathrm{hp.001}$, quits the program, and starts it again two days later in the same directory, the next saved file:will be called $\mathrm{hp.002}$. The autoincrement process would continue through file hp.999, then would wrap back to file hp.000.

\subsection{Output -- Printing, Hardcopy, Importing Plots into Word Processors (Plot Screen)} Use the "Output" button (or type 'O') to send the plot to a file. Currently, PP produces only HPGL (Hewlett-Packard Graphics Language) files. However, future versions of the code may support PostScript. HPGL files are understood by most PC-based applications, and can be imported into most CAD programs and all versions of WordPerfect $\geq 5.0$.

There are three ways to obtain hardcopy plots:

(1) Send output directly to a plotter or printer from within PP;

(2) Create plot files with the Output button, quit PP, then print the plot files from the DOS prompt;

(3) quit PP and import the plot files (e.g. hp.000, hp.001) into a word processor or CAD program.

The first method works with an HP Laserjet III or 4 printer, a compatible laser or inkjet printer with an HPGL emulation mode, or a true HP plotter (7400 series or later). Use the File dialog (section 5.8) to select "Graphics format= HPGL + laserjet codes", and set the "output Filename" to be PRN or the port appropriate for your hardcopy device (e.g. LPT1, COM1, COM2). Leave the File dialog and click on the Output button; the screen will update slowly as the individual HPGL commands are written to the device. In rare circumstances, the printer may "time out" while the file is being printed, but the timeout should be intercepted by PP's critical error handler.

The second method works with the same devices as the first method. At the DOS prompt, one types print $\mathrm{hp} .000 \hookleftarrow$ to print the file hp.000. The "Graphics format= HPGL + laserjet codes" selection creates files that contain the codes necessary to put most HP-compatible printers into plotter mode, as well as the codes to put the printer back in PCL mode and eject the page at the completion of the plot. These codes are on the very first and very last lines of the file, are preceded by ASCII 27 (the escape character, a left-pointing arrow), and can be removed with an ASCI editor.

The third method requires one to "import" the file into a program, such as a word processor. This method is the most flexible, since word processors and CAD programs have drivers for virtually all plotters and printers, and one can often edit or annotate the plot with the program.

For WordPerfect for Windows, best results are obtained when one chooses the "HPGL WpWin 5.1/5.2" format from the File dialog. In version 5.2, the "Graphics-Figure-Retrieve" dialog is then used to import the file. Files created with the "HPGL+laserjet codes" and "pure HPGL (MS Word)" options can still be imported, but it is best to first convert them to * wpg files with the "Graphenv" utility, using the /w switch to ensure thin 
lines, e.g.:

\section{Iwpelgraphenv hp.000 hp.wpg /w}

To produce HPGL files for MS Word, use the "pure HPGL (MS Word)" format in the "File options" menu. However, one can import a file produced with the "HPGL+laserjet codes" option by first editing the "SC command" in the output file. This command will be on the 1st or 2nd line, and will be similar to:

\section{IN; RO9 0 IP ; IW; SC0, 7990, 0,5990;FT1;PA; SP1;}

the 7990 and 5990 refer to the maximum $x$ and $y$ coordinates for the plot (these numbers might be different on your system). To have MS Word import the plot, increase these numbers by a factor of about 1.25 -- e.g., increase 7990 to 9980 , and 5990 to 7480 . If the increase is not made, the MS Word import filter will chop off the right and upper margins. Remember that these changes should be unnecessary if the "pure HPGL (MS Word)" option is chosen from the file menu in the plot screen.

To import an HPGL file in MS Word for Windows, one typically selects "insert" from the top menu bar, then "picture", then one uses the file dialog to select the file name. Once the picture is imported, double-clicking on it will bring up a picture editor that allows one to add text, draw lines, etc. on the figure. This picture editor is called MS Draw; while it comes with Word, it may not be installed on all systems.

If MS Word cannot read the HPGL files, it may be necessary to install the MS graphics filters. These filters are independent of MS Draw, and many system administrators DO NOT install them by default. With MS Word for Windows, the filter installation is performed via the setup program (typically on the installation floppy disc). Choose the custom installation, and unselect every choice except installation of the graphics filters, then click on the filters button and unselect every filter except the HPGL filter. To "unselect" an option, click on it with the mouse to remove the cross from the selection box.

Some applications may require that the HPGL files be converted into PostScript. In such a case, the public domain conversion program hp2xox (widely available on internet) can be used to make the conversion. For example, the DOS version could be used to convert the HPGL file hp. 000 to an encapsulated PostScript file out.ps via:

hp2xados $-\mathrm{m}$ eps $-\mathrm{f}$ out.ps hp.000

out.ps can then be imported into a word processor and embedded in a document. 


\section{The FF Font Editor}

The program ff .exe can be used to edit PP's "external" font (figure 20). Copy the file external . fnt to external .bak and test . fnt, then start FF. In FF, click on "Retrieve" (or type ' $R$ ') to edit test. fnt. The screen will display an exclamation point, '!', which is ASCII character number 33, the first printable character. Clicking repeatedly on the "Next" button (or typing ' $\mathrm{N}$ ') will advance through the successive ASCII characters:

"\#\$\%\&'( )*+, - . / 01223456789 : ; < = ? \& A B C D E F G and so on.

To create a new character, or add a line to a character, move the mouse cursor to the desired position and click the left mouse button. A green "rubber band line" forms, with one end attached to the position of the last left click, and the other attached to the mouse cursor; the program is now in drawing mode. Each subsequent left click tacks down the "rubber band" to that position on the screen. If you make a mistake, press the Escape key to detach the last point; pressing Escape repeatedly will eventually "erase" the line. To complete the line, and leave drawing mode, click the right mouse button. The completed line turns from green to yellow. Each character may have up to 32 polylines, each with up to $32 \mathrm{x}, \mathrm{y}$ pairs (or 31 line segments per polyline).

To edit an existing line, move the mouse cursor near the line and click the right mouse button. The mouse cursor will attach itself to the end of the nearest line; the line can be deleted by repeatedly pressing the Escape key. The program will remain in drawing mode until one either deletes all the points on the line, or clicks the right mouse button.

Note the light cyan buttons on the right side of the screen. The "Quit" button exits the program. Clicking on the "Grid" button toggles the display of the positioning grid on and off. Toggling the "snAp" button ON forces the mouse pointer to move in 8 pixel increments on the grid. The "Retrieve" button pulls in the file test. Int for editing; the "Save" button saves the same file. "Next" and "Previous" move forward and backward in the ASCII character set (the corresponding ASCII character is displayed at the bottom of the screen). The "Zero line" button allows one to change the position of the $y$-axis zero line. The default position is at the bottom of the screen, but the zero line should be moved up for characters with descenders, such as $y, p, g$, and $j$.

When done editing, be sure to save the altered file (by clicking on "Save" or typing 'S'), exit FF (by clicking on "Quit" or typing 'Q'), and copy test. fnt to external .fnt.

The FF editor can also create custom symbols (markers) to plot at the nodes of the reaction path curves (figure 21). The eight characters from ASCII 128 to 135 in external . fnt are reserved for custom symbols (in the standard PC character set, these are the letters Ç,ü,ê,âa,a,à,å, and ç). When invoked, PP scans these characters to see if they have been defined (that is, if they contain any lines). If defined, the custom symbols are added to the selections available from the variables and plot screens. 


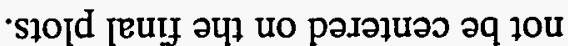

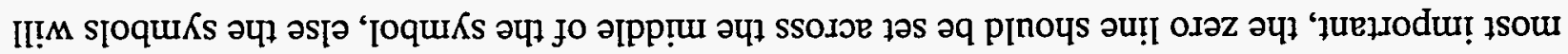

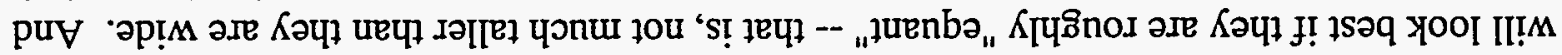

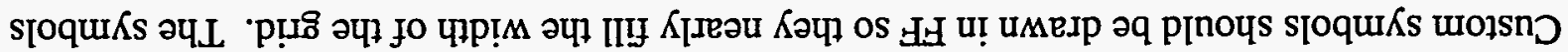

-8นu!̣pa Iof

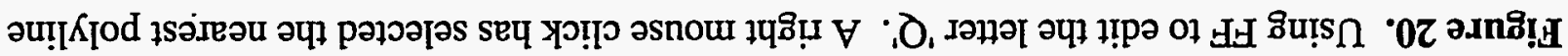

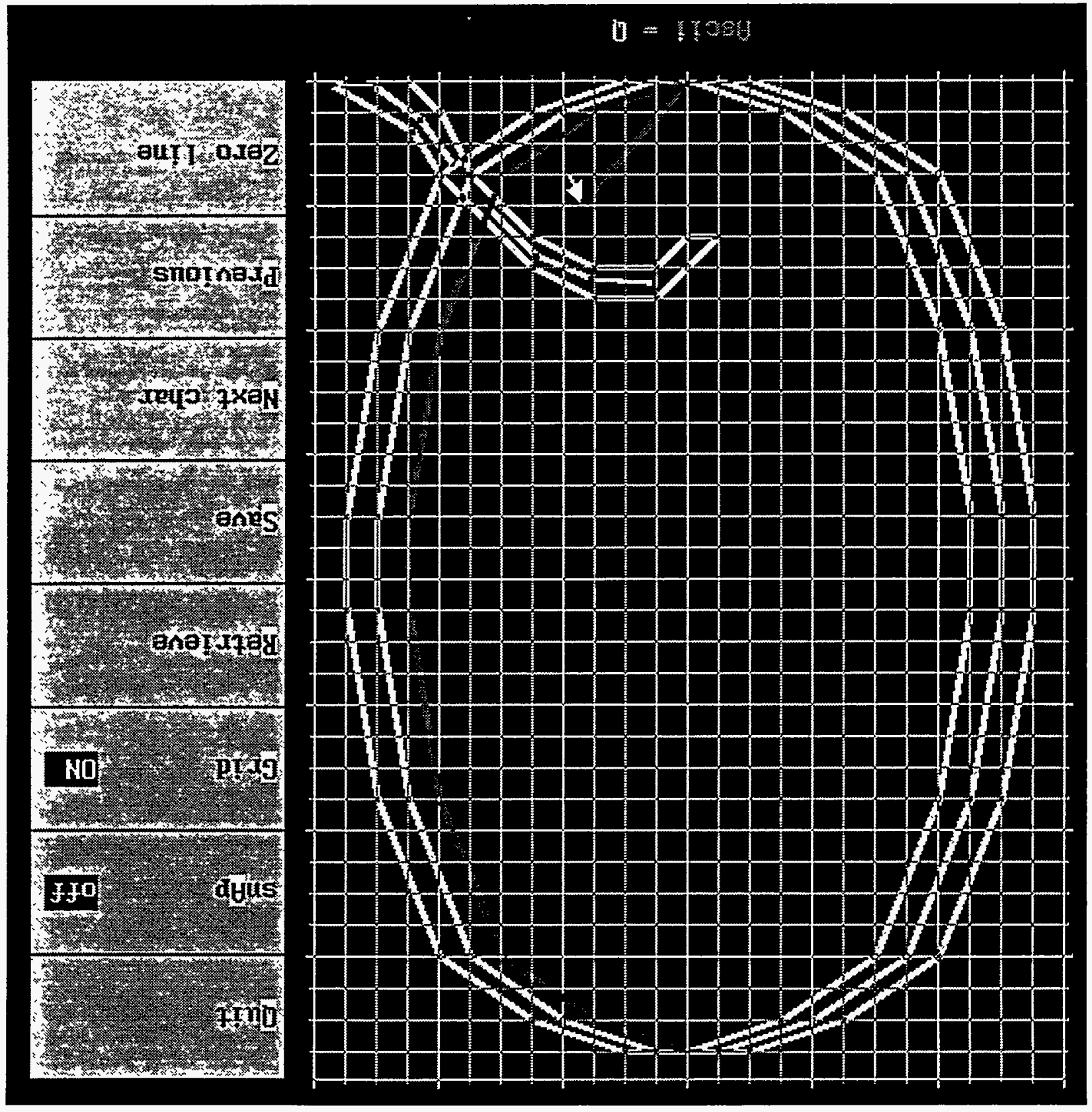




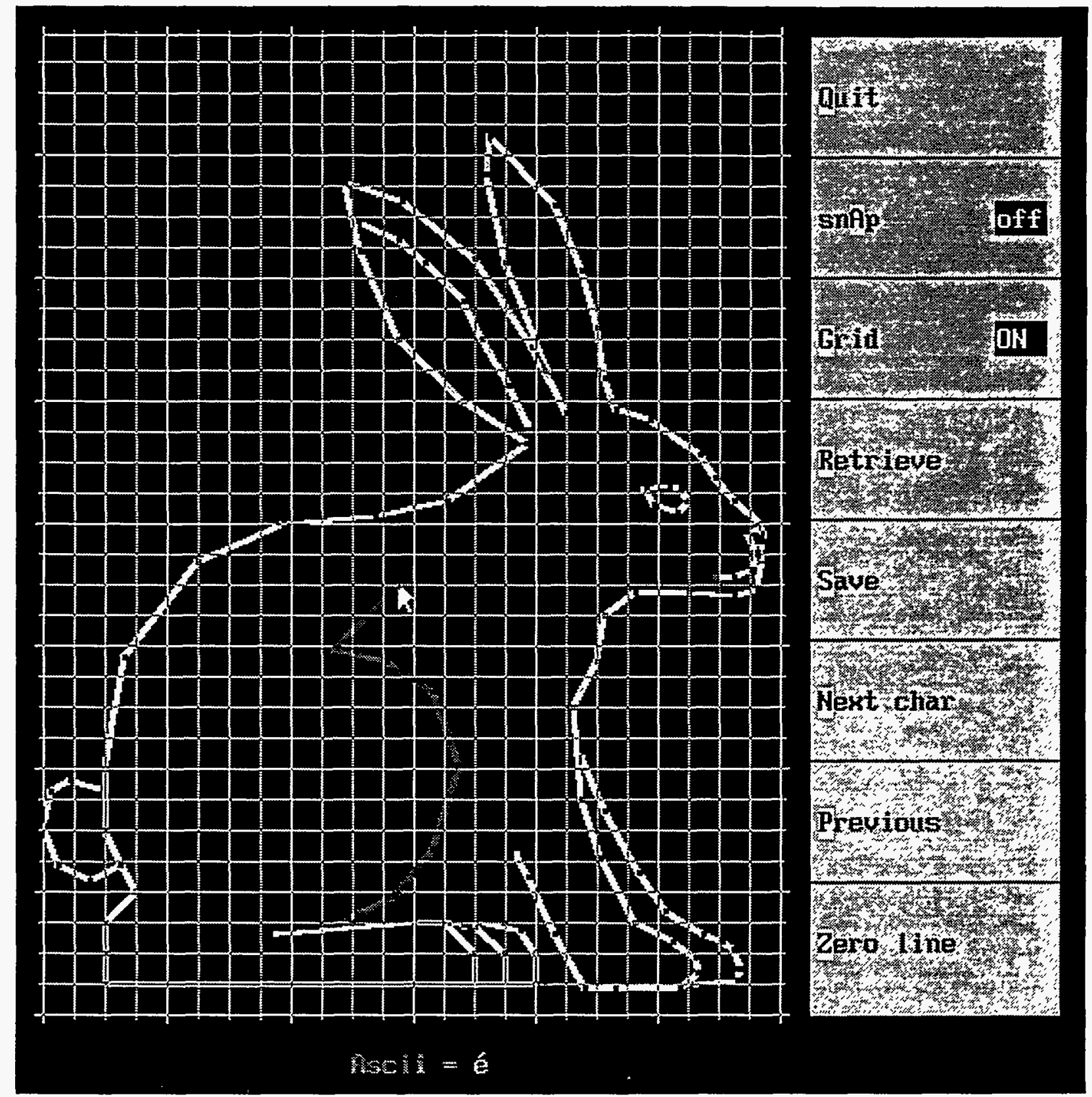

Figure 21. Using FF to edit a custom symbol in the font file (external . fnt).

There are two significant differences between custom symbols and the ordinary symbols (such as circles and squares) built into PP. First of all, the custom symbols plot larger; it is assumed that the larger size will be needed to show the details of the symbol design (figure 22). Second, PP generally does not draw lines through the custom symbols, since the lines would obscure the details. However, connecting lines are plotted between the symbols wherever possible. 


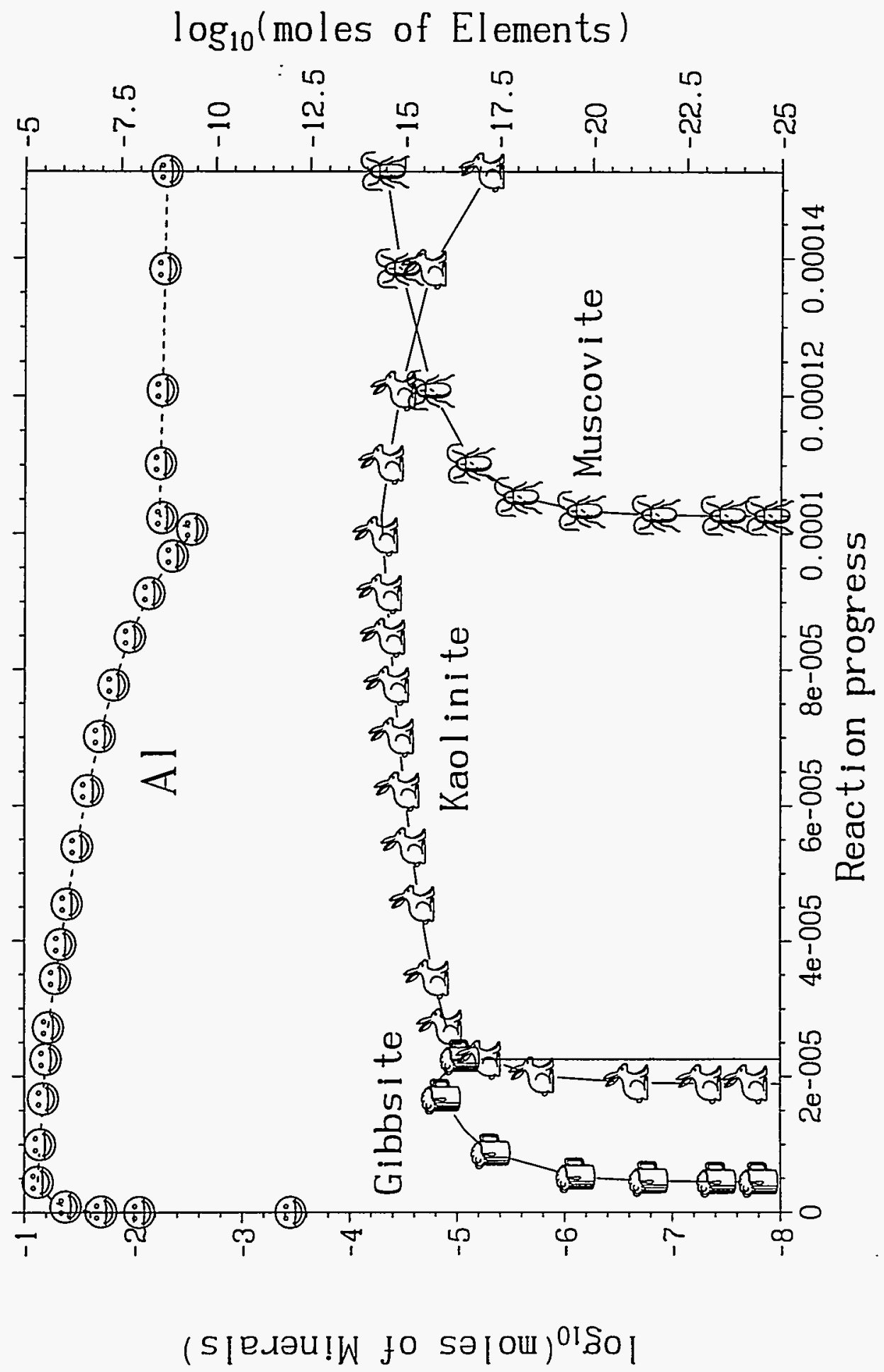

Figure 22. Using custom symbols in a plot. 


\section{Implementation Details}

$\mathrm{PP}$ is written in C, and compiled with Symantec C version 6.0 , using the -4 - $\mathrm{mx}-\mathrm{f}$ witches and no optimization. With comments, there are approximately 10000 lines of source code. The screen graphics primitives, such as line-drawing, pixel plotting, bitblt, and character printing, are provided by the FlashTek "Flash Graphics" libraries. The executable contains a DOS extender, so that when the program is invoked from the DOS prompt, the CPU is placed in protected mode with a 32-bit, flat address space.

Nearly all of PP's arrays are allocated dynamically. Thus, PP will adjust its memory requirements to the number of variables used by the EQ6 run that produces the hwsdata file. When data are imported, many of the arrays are reallocated to provide consistency in the graphics routines. The functions that display the help_pp and data 0 * files read and allocate memory for these files with each invocation, and deallocate the memory at the completion of the function call. Thus each help or search call must re-read the files from disk; this process may seem inefficient, but allows PP to run on minimal systems, and in practice the delays are small.

The PP source code uses EQ6 notation for arrays whenever possible. For example, the PP array of mineral names is UMIN, and the array of "moles of total elements in aqueous solution" is called MTEAQ. However, if an EQ6 data array (as opposed to name array) has dimension N, the corresponding PP array generally has dimension $\mathrm{N}+1$, where the extra dimension is the step number. The PP data arrays are declared as, e.g., double * MTEAQ.

PP contains numerous algorithms for editing text lines, drawing stroked fonts, parsing species formulae, clipping, and choosing axis ranges and increments. The editing, font, parsing and axis algorithms were developed independently by the author. The clipping functions are substantially modified versions of the NCSA Telnet routines. The NCSA routines are not copyrighted and are derived from the Liang-Barsky algorithm (Liang and Barsky, 1984). The NCSA code follows the published algorithm, maintaining the same variable names and changing only from Pascal to C syntax. We gratefully acknowledge the generosity of NCSA in making these routines available.

Currently, the most serious shortcoming of PP is the slowness of the text operations. Overall, the FlashTek graphics routines are very good. However, the FlashTek text routines preserve the background behind each character; this feature is quite ingenious, but is undesirable for PP and adds enormous overhead. It is estimated that the FlashTek bit-mapped text graphics are approximately 20 times slower than the equivalent MS Windows routines for modern, accelerator-based graphics cards. This slowness is manifest in high resolution modes (1024 by 768 pixels or more), where it may take a second to paint the screen on an ISA-bus 486 computer. In addition, many of the Symantec string functions are $C$ compiled for the 386 , and could be greatly improved by translation to assembly language, or recompilation for the Pentium. 


\section{Conclusions}

PP provides a simple but flexible interface to EQ6, allowing rapid analysis of reaction path runs. Simple plots of one data type (e.g., mineral amounts or solution composition) require two mouse clicks -- one to select the data, and one to plot. The program is designed for rapid navigation through the variable list, with a minimal amount of detail to confuse the user. While the user is free to select and customize axis ranges, most scaling decisions take place transparently, without user intervention. PP accommodates the specialized needs of reaction path plots, and simplifies the process of placing and editing labels and curve characteristics. Compatibility with other DOS and Windows programs is provided by a simple, standardized graphics format (HPGL), and the ability to export and import spreadsheet and ASCII data files. Useful changes for future versions of PP would include: improved speed of text operations; and migration to a richer operating system, such as MS Windows. 


\section{References}

Bruton, C.J. and Shaw, H.F. (1988) Geochemical simulation of reaction between spent fuel waste form and J-13 water at $25^{\circ} \mathrm{C}$ and $90^{\circ} \mathrm{C}$, in: M.J. Apted and R.E. Westerman (eds.)

Scientific Basis for Nuclear Waste Management, Materials Research Society, pp 485-494.

Criscenti, L.J. and Arthur, R.C. (1991) The calculated effects of isothermal boiling on tuff-water interactions, Radiochimica Acta v 52, pp 513-517.

Gardiner, M.A.; Alcorn, S.R.; Myers, J. and Givens, C.A. (1989) Modeling simple cement-water systems using the speciation/solubility/reaction path computer codes EQ3NR/EQ6, with specific application to nuclear waste repositories, in: D.L. Miles (ed.) Water-Rock Interactions WRI-6, pp 235-238.

Liang, Y.-D. and Barsky, B.A. (1984) A new concept and method for line clipping, ACM Transactions on Graphics v 3, pp 1-22.

Wolery, T.J. (1992) EQ3/6, A Software Package for Geochemical Modeling of Aqueous Systems: Package Overview and Installation Guide (Version 7.0). UCRL-MA-110662 PT1, Lawrence Livermore National Laboratory, Livermore, CA 94551, 66 pp. 


\section{Definitions, Copyrights and Trademarks}

386, 486DX, 486SL 32-bit Microprocessors, trademarks of Intel Corporation

ASCII

BIOS

bitblt

DOS

$\mathrm{EQ} 3 / 6, \mathrm{EQ6}$

FlashTek

Hewlett-Packard:

laserjet, $1200 \mathrm{C}$,

7400 series

HPGL

IBM

MB

Microsoft:

MS DOS

MS Draw

MS Word

notepad

NCSA

PCL

Pentium

pixel

PostScript

RAM

reaction path

ROM

Symantec

VESA

VGA

Windows

WordPerfect
American Standard Code for Information Interchange

Basic Input-Output System, a series of machine-code programs for communicating with disk drives and peripherals, located in read-only memory (ROM)

A block move of pixels

Disk Operating System

Equilibrium/reaction path codes copyrighted by University of California Trademark of FlashTek, Inc.

Trademarks of Hewlett-Packard Corp.

Hewlett-Packard Graphics Language, a vector plotter language

Trademark of International Business Machines Corp.

Megabyte, or $2^{20} 8$-bit bytes

Trademarks of Microsoft, Inc.

National Center for Supercomputing Advances

Printer Command Language (Hewlett-Packard)

32/64-bit microprocessor, trademark/copyright of Intel Corp.

A single $(x, y)$ addressable point on a graphics screen

A page-description language, trademark of Adobe Corp.

Random Access Memory (electronic memory)

A record of the molar amounts and activities of species and components

in the aqueous, solid and gaseous phases of a chemical system, as

components are incrementally added or removed.

Read-Only Memory (electronic memory)

Trademark of Symantec Corp.

Video Electronics Standards Association

Video Graphics Array, trademark of IBM Corp.

Trademark of Microsoft, Inc.

Trademark of WordPerfect Corp. 


\section{APPENDIX: EQ6 Modifications for Creating HWSDATA}

PP reads binary data files created by a modified version of EQ6. EQ6 was written by Thomas J. Wolery of Lawrence Livermore National Laboratory (LLNL), and is copyrighted by that author for the Department of Energy and the Regents of the University of California. The process for obtaining the EQ6 source is described in the EQ3/6 documentation (Wolery, 1992). This appendix describes the changes one must make to EQ6 to produce the hwsdata binary file, but does not contain any EQ6 code. It is assumed that EQ6 will subsequently be recompiled with Lahey FORTRAN or a compatible compiler.

The following function should be appended to the EQ6 source:

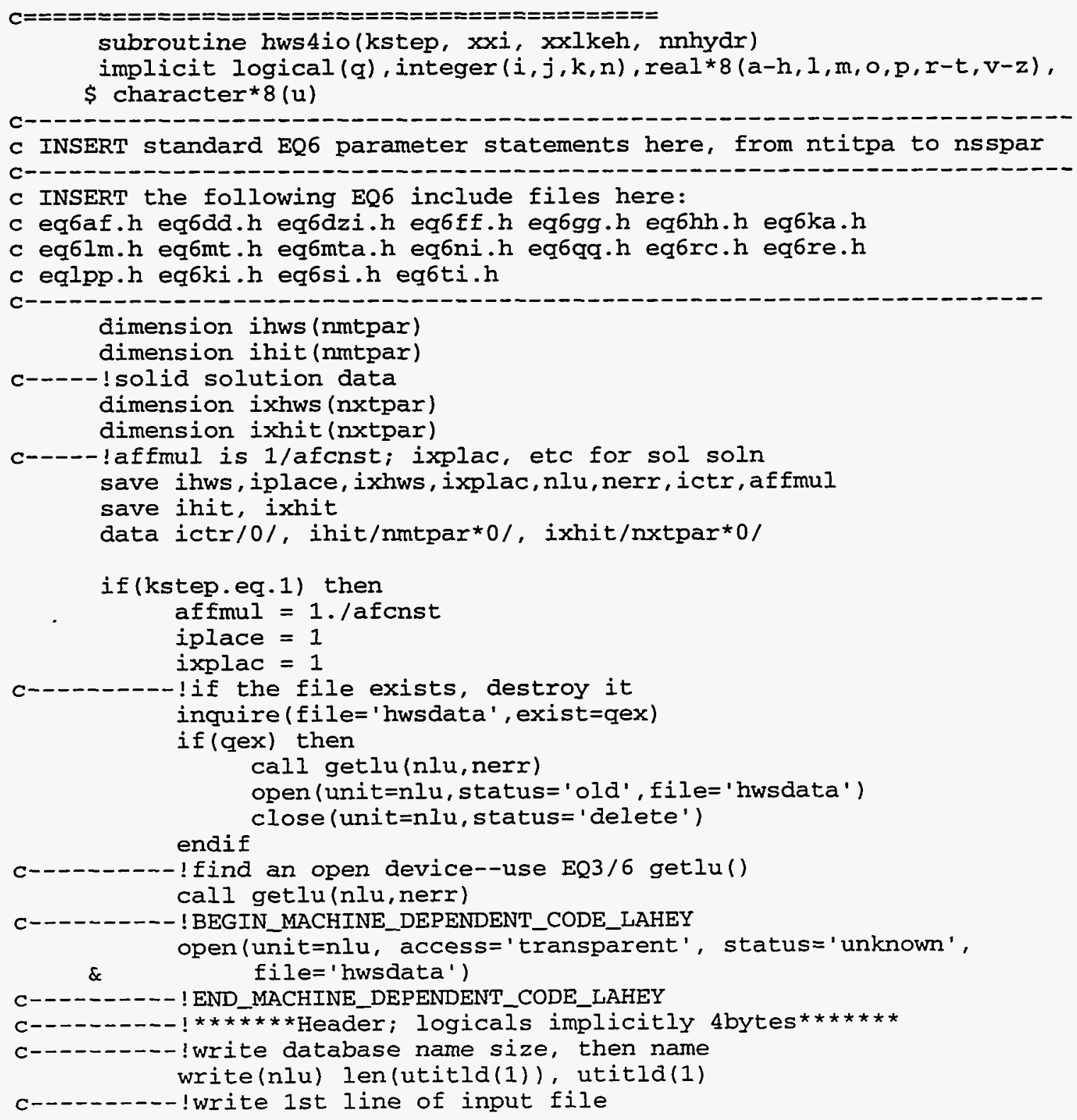


write(nlu) len(utitll(1)), utitll(1)

write(nlu) qdump, qnochb

c-------! Write number of active...

write(nlu) kct, jgt, jmt, jst, jxt

c-n---- ! Write the \# reactants and the reactant names

write(nlu) nrct

do $20 i=1$, nret

if (jcode( $i)$.eq.1) then

else

write(nlu) usolx(nrndex(i))

endif

write(nlu) ureac(i)

c-------!Element, species, mineral names for $\log Q / K$, gas names

do $21 i=1$, nct

21 continue

if(jcflag(i).gt.0) write(nlu) uelem(i)

do $22 i=1$, nst

22 continue

if(jsflag(i).le.0) write(nIu) uspec(i)

do $23 i=1$, nmt

if(jmflag(i).le.0) write(nlu) umin(i)

23 continue

do $24 i=1, \mathrm{nxt}$

24 continue if(jxflag(i).le.0) write(nlu) usolx(i)

do $25 i=1$, ngt

25 continue if(jgflag(i).le.0) write(nlu) ugas(i)"

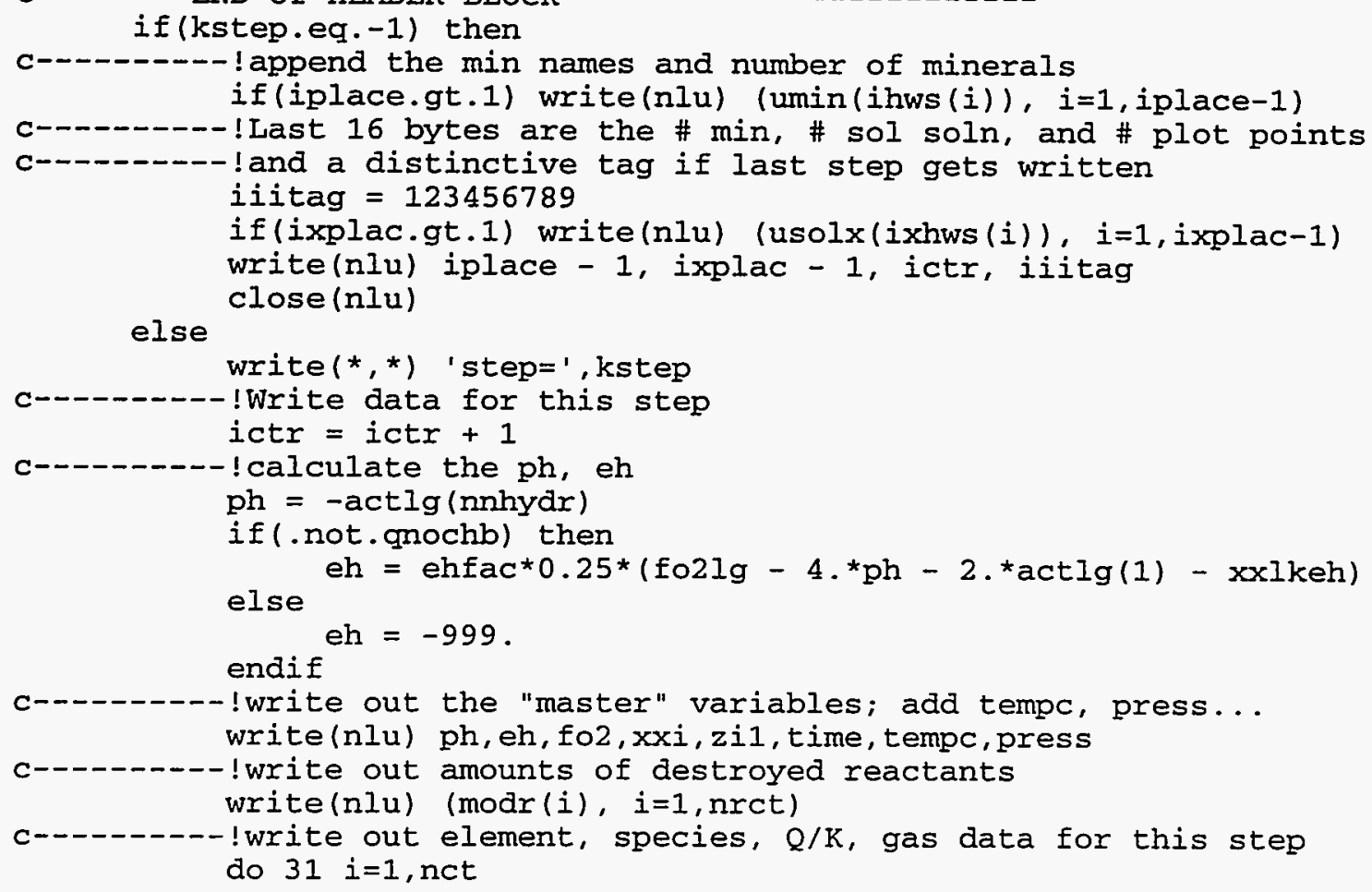




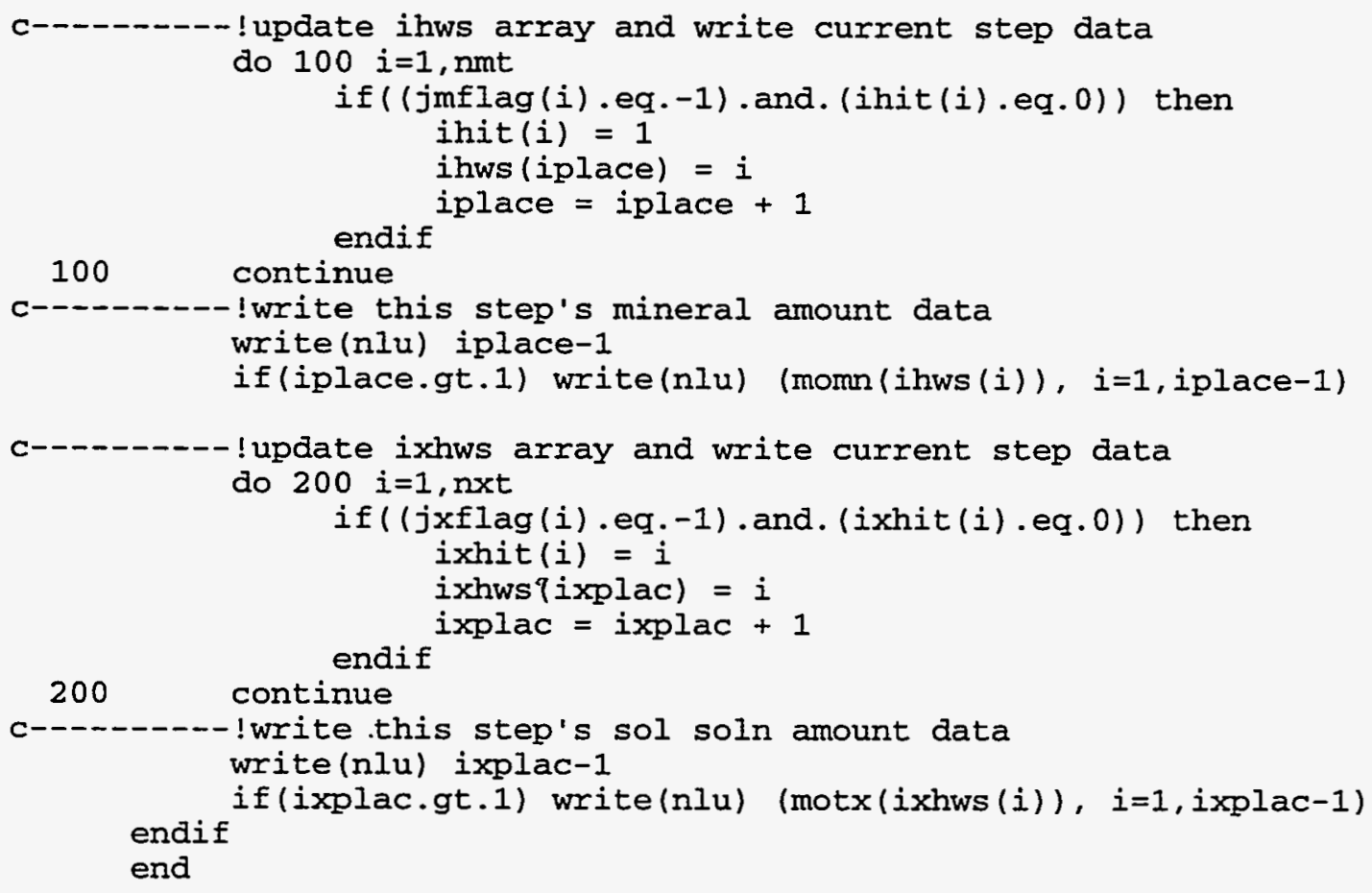

Place the following statement in the EQ6 path () routine, immediately after the statement at label 20 ( 20 kstep=kstep+1):

call hws4io(kstep, xi, xlkeh, nhydr)

then place the following 2 statements in path ( ), between the "999 continue" and "end" statements:

call hws4io(kstep, xi, xlkeh, nhydr)

call hws 4 io $(-1, x i, x l k e h$, nhydr)

and recompile EQ6 according to the instructions in the EQ3/6 release notes. 


\section{Distribution}

T.J. Wolery, L-219

Lawrence Livermore National Laboratory

P.O. Box 808

Livermore, CA 94550

D.R. Janecky, INC 7

Los Alamos National Laboratory

MS J514

Los Alamos, NM 87545

W.M. Murphy

Southwest Reasearch Institute

6220 Culebra Rd.

POD 28510

San Antonio, TX 78228-0510

$\begin{array}{ll}\text { MS0750 } & \text { H.R. Westrich, 6118 (1) } \\ \text { MS0750 } & \text { H.W. Stockman, 6118 (1) } \\ \text { MS0750 } & \text { J.L. Krumhansl, 6118 (1) } \\ \text { MS0727 } & \text { M.S.Y. Chu, 6622 (1) } \\ \text { MS1328 } & \text { J.W. Garner, 6342 (1) } \\ \text { MS1341 } & \text { L.H. Brush, 6348 (1) } \\ \text { MS1347 } & \text { L.A. Dawson, 7582 (1) } \\ & \\ \text { MS0899 } & \text { Technical Library, 13414 (5) } \\ \text { MS0619 } & \text { Technical Publications, 13416 (1) } \\ \text { MS1119 } & \text { Document Processing, 7613-2 (10) } \\ & \text { For DOE/OSTI } \\ \text { MS9018 } & \text { Central Technical Files, 8523-2 (1) }\end{array}$

